UCB-PTH-09/02

\title{
Dark Matter Signals from Cascade Annihilations
}

\author{
Jeremy Mardon, Yasunori Nomura, Daniel Stolarski, and Jesse Thaler \\ Berkeley Center for Theoretical Physics, Department of Physics, \\ University of California, Berkeley, CA 94720 and \\ Theoretical Physics Group, Lawrence Berkeley National Laboratory, Berkeley, CA 94720
}

\begin{abstract}
A leading interpretation of the electron/positron excesses seen by PAMELA and ATIC is dark matter annihilation in the galactic halo. Depending on the annihilation channel, the electron/positron signal could be accompanied by a galactic gamma ray or neutrino flux, and the non-detection of such fluxes constrains the couplings and halo properties of dark matter. In this paper, we study the interplay of electron data with gamma ray and neutrino constraints in the context of cascade annihilation models, where dark matter annihilates into light degrees of freedom which in turn decay into leptons in one or more steps. Electron and muon cascades give a reasonable fit to the PAMELA and ATIC data. Compared to direct annihilation, cascade annihilations can soften gamma ray constraints from final state radiation by an order of magnitude. However, if dark matter annihilates primarily into muons, the neutrino constraints are robust regardless of the number of cascade decay steps. We also examine the electron data and gamma ray/neutrino constraints on the recently proposed "axion portal" scenario.
\end{abstract}




\section{Introduction}

Recent observations by PAMELA [1] and ATIC [2] strongly suggest a new primary source of galactic electrons and positrons. Three leading interpretations of the PAMELA/ATIC excesses are astrophysical sources [3], decay of dark matter [4], and annihilation of dark matter [5, 6, 17, 8, 9]. While the current PAMELA/ATIC data cannot distinguish between these possibilities, one expects that the correct scenario will ultimately be determined with the help of complementary data from synchrotron, gamma ray, and neutrino telescopes, as well as collider and direct detection experiments.

One piece of data that points toward an annihilation interpretation is the WMAP Haze [10], an apparent excess of synchrotron radiation coming from the galactic center. Dark matter annihilation into charged particles is uniquely positioned to explain the Haze [11, 12. If $n$ is the dark matter number density near the galactic center, then the synchrotron signal for dark matter annihilation scales like $n^{2}$, while the signal for dark matter decay scales only as $n$. (Astrophysical signals also roughly scale like $n$.) Given the normalization of the PAMELA/ATIC excess, the $n^{2}$ scaling is favored to explain the size of the Haze anomaly [13].

On the other hand, the same $n^{2}$ versus $n$ logic implies that the dark matter annihilation interpretation is more strongly constrained by the absence of gamma ray or neutrino excesses from the galactic center. While these constraints are dependent on the Milky Way dark matter halo profile, there are already strong bounds on the annihilation interpretation for strongly peaked halos [14, 15, 16, 17, 18]. Therefore, it is worth exploring dark matter annihilation scenarios in detail to understand how robust the tension is between explaining PAMELA/ATIC/Haze and satisfying other bounds.

Given the absence of anti-proton [19] or gamma ray [20, 21, 22] excesses, the dark matter annihilation scenarios favored to explain PAMELA/ATIC involve annihilation into electrons and muons. However, dark matter need not annihilate into leptons directly. There are a variety of "cascade annihilation" models where dark matter annihilates into light resonances which in turn decay into electrons or muons. These light resonances can lead to nonperturbative enhancements [23, 24] of the dark matter annihilation rate in the galactic halo, providing the large boost factors necessary to explain PAMELA/ATIC [5, 6, 7]. Also, annihilation into light fields gives a kinematic explanation for why dark matter annihilation preferentially yields light leptons [12, 6, 7]. Previous studies of cascade annihilation models appear in Refs. [8, 16].

In the present context, these cascade annihilation scenarios are interesting because they have the potential to explain PAMELA/ATIC while weakening constraints from gamma rays, as measured by atmospheric Cerenkov telescopes like H.E.S.S. [20, 21, 22]. The reason is that gamma ray experiments are directly sensitive to the primary injection spectra, and cascade annihilations yield softer and smaller injection spectra of gamma rays from final state radiation (FSR). 
PAMELA/ATIC sees electrons and positrons through the filter of charged cosmic ray transport, a process which introduces large uncertainties. Considering also the uncertainties in the highest energy ATIC data, we find that softer spectra of primary leptons can still explain the PAMELA/ATIC excesses.

For cascade annihilations that terminate in muons, there is also an irreducible source of galactic neutrinos, which can be observed as an upward-going muon flux on earth, for example, by water Cerenkov detectors like Super-Kamiokande (Super-K) [25]. While cascades soften the neutrino spectrum, we will see that the final constraints from neutrinos are rather insensitive to the number of cascade steps, and may provide the most robust bound on muon cascade scenarios.

The organization of this paper is as follows. In the next section, we define our framework for analyzing signals of dark matter through cascade annihilations, with details of the cascade energy spectra given in Appendix A. In Section 3, we find the best fit dark matter masses and annihilation cross sections for various cascade scenarios given the PAMELA/ATIC data. We consider H.E.S.S. gamma ray bounds from FSR in Section 4 and Super-K neutrino bounds in Section 5. In Section 6, we study a particular cascade annihilation scenario called the axion portal [7], and present a less constrained "leptonic" version in Appendix B. Conclusions are given in Section 7.

\section{Cascade Annihilations}

If dark matter is a thermal relic, then it will have at least one annihilation mode into standard model fields, since in the early universe, the dark matter annihilation channels keep dark matter in thermal equilibrium with the standard model until freezeout. However, dark matter need not annihilate into standard model particles directly; it can annihilate into new (unstable) resonances which in turn decay into standard model fields. As long as the new resonances are sufficiently broad, then dark matter will be in close enough thermal contact with the standard model for a freezeout calculation to be valid.

These "cascade annihilations" can occur in one or more steps, as shown in Figure 1, and there are a variety of motivations for such cascade scenarios. Since the direct coupling between dark matter and the standard model can be small while still achieving the desired thermal relic abundance, such models can have reduced direct detection cross sections compared to generic weakly interacting massive particles (WIMPs) [26]. Cascade annihilations can arise whenever new light resonances have large couplings to dark matter, such as in exciting dark matter [27]. In the context of PAMELA/ATIC, there has been interest in using light resonances to provide large enhancement to the galactic annihilation rate through the Sommerfeld effect or bound state (WIMPonium) formation [5, 6, 7]. These light resonances can also explain the lepton-richness of dark matter annihilation through kinematic thresholds [6, 7]. 


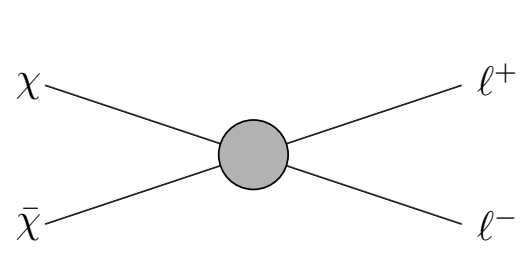

direct

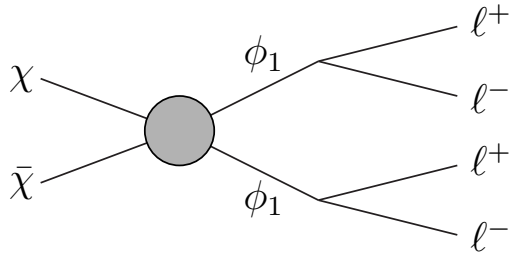

1 step

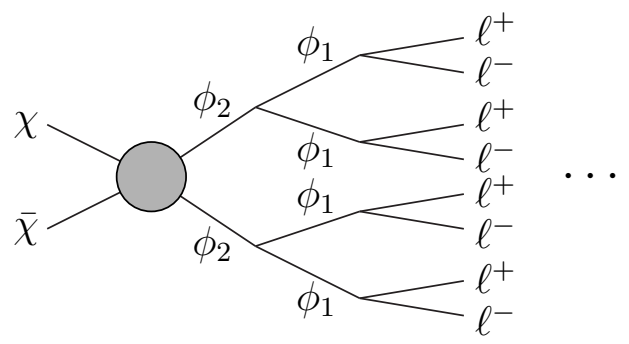

2 step

Figure 1: For an $n$-step cascade annihilation, dark matter $\chi$ annihilates into $\phi_{n} \phi_{n}$. The cascade annihilation then occurs through $\phi_{i+1} \rightarrow \phi_{i} \phi_{i}(i=1, \cdots, n-1)$, and in the last stage, $\phi_{1}$ decays into standard model particles. The figure represents the cases where $\phi_{1} \rightarrow \ell^{+} \ell^{-}$.

Cascade annihilations give softer primary spectra for the annihilation products than direct annihilation. As reviewed in Appendix A, for (scalar) cascade annihilations involving well-separated kinematic scales, the approximate primary spectra can be calculated using a simple convolution formula. The energy spectra are conveniently parametrized in terms of the energy fraction $x_{n} \equiv E_{n} / m_{\mathrm{DM}}$, where $E_{n}$ is the final state energy after the $n$-th step of the cascade, and $m_{\mathrm{DM}}$ is the dark matter mass. If $d \tilde{N} / d x_{0}$ is the normalized primary spectrum from direct annihilation, then the normalized primary spectrum from a 1-step annihilation $d \tilde{N} / d x_{1}$ is

$$
\frac{d \tilde{N}}{d x_{1}} \approx \int_{x_{1}}^{1} \frac{d x_{0}}{x_{0}} \frac{d \tilde{N}}{d x_{0}} .
$$

This convolution formula can be iterated to obtain the normalized primary spectrum for an $n$-step cascade scenario. Eq. (1) is also reasonably representative of non-scalar cascades, since indirect detection measurements are unpolarized.

While cascade annihilations give softer spectra, they typically yield a higher multiplicity of annihilation products. The final primary spectrum $d N / d x_{n}$ must take into account the multiplicity of annihilation products per dark matter annihilation, and in a typical $n$-step cascade, the final state multiplicity scales like $2^{n}$.

An important exception, however, is in cases involving singularities, where cascade annihilations yield both a softer spectrum and a lower multiplicity. For example, FSR from charged leptons has a collinear singularity that is regulated by the lepton mass. Integrating over the singularity gives a photon spectrum $d N_{\gamma} / d x$ that is proportional to $\ln Q / m_{\ell}$, where $Q$ is the total energy available for radiation. For direct annihilation $Q \simeq 2 m_{\mathrm{DM}}$, while for cascade annihilations $Q \simeq m_{1}$, where $m_{1}$ is the mass of the resonance in the last stage of the annihilation $\phi_{1} \rightarrow \ell^{+} \ell^{-}$. Light enough $\phi_{1}$ fields can give a dramatic reduction in the $\ln Q / m_{\ell}$ factor and thus the FSR photon yield. 
In this paper, we only consider cascade annihilations that terminate in electrons or muons. The same analysis, however, could be repeated for charged pions or taus by changing the direct annihilation spectra. We expect the results for an $n$-step charged pion cascade to be similar to an $(n+1)$-step muon cascade. Cascades involving taus will face stronger gamma ray bounds because of the $O(1)$ fraction of $\pi^{0} \mathrm{~s}$ in tau decays. For simplicity, we only show plots for direct, 1-step, and 2-step cascades.

\section{PAMELA/ATIC Spectra}

The PAMELA satellite experiment [28] observed an anomalous source of galactic positrons in the energy range 10-100 GeV through a measurement of the positron fraction $\Phi_{e^{+}} /\left(\Phi_{e^{+}}+\Phi_{e^{-}}\right)[1]$. The ATIC balloon experiment [29] is not capable of charge separation, but observed a peak in the total electron plus positron flux between $100-1000 \mathrm{GeV}$ in a $\left(\Phi_{e^{+}}+\Phi_{e^{-}}\right)$measurement [2]. Intriguingly, both excesses can be described by a single new source of galactic electrons and positrons, and here we study the goodness of fit for dark matter cascade annihilations. The primary electron/positron spectra for $n$-step electron and muon cascade annihilations are given in Appendices A.1 and A.2. Once the primary spectrum is known and a dark matter halo profile assumed, we can propagate the electrons and positrons through the Milky Way and compare with the PAMELA/ATIC data.

We follow the analysis of Ref. [30], which assumes that galactic electrons and positrons can be described by a diffusion-loss process. In the turbulent galactic magnetic fields, electrons/positrons diffuse within a fiducial region around the galactic disk and escape the galaxy outside that region. An energy loss term incorporates the physics of inverse Compton scattering (ICS) and synchrotron radiation. Dark matter annihilation is represented by a source term proportional to the square of the dark matter halo density. Since the energy loss time is much shorter than the age of the galaxy, the electron/positron system is assumed to be in steady state.

Taking $\psi_{e^{-}}(\vec{x}, E)$ to be the galactic electron number density per unit energy, the diffusion-loss equation is

$$
K_{0} \varepsilon^{\delta} \nabla^{2} \psi_{e^{-}}(\vec{x}, E)+\frac{\partial}{\partial \varepsilon}\left(\frac{\varepsilon^{2}}{\tau_{E}} \psi_{e^{-}}(\vec{x}, E)\right)+q(\vec{x}, E)=0
$$

where $\varepsilon=E / \mathrm{GeV}, K_{0}$ and $\delta$ parametrize the (energy dependent) diffusion, $\tau_{E}$ is a characteristic energy loss time, and $q(\vec{x}, E)$ is the electron source term for dark matter annihilations. The same equation also holds for the positron number density per unit energy $\psi_{e^{+}}(\vec{x}, E)$. The electron/positron densities $\psi_{e^{-} / e^{+}}(\vec{x}, E)$ are assumed to have vanishing boundary conditions on the surface of a cylinder of height $2 L$ and radius $R$. We consider the three benchmark models from Ref. [30], which are summarized in Table 1. 


\begin{tabular}{c|ccccc} 
& $R(\mathrm{kpc})$ & $L(\mathrm{kpc})$ & $K_{0}\left(\mathrm{kpc}^{2} / \mathrm{Myr}\right)$ & $\delta$ & $\tau_{E}(\mathrm{sec})$ \\
\hline MED & 20 & 4 & 0.0112 & 0.70 & $10^{16}$ \\
M1 & 20 & 15 & 0.0765 & 0.46 & $10^{16}$ \\
M2 & 20 & 1 & 0.00595 & 0.55 & $10^{16}$
\end{tabular}

Table 1: Diffusion-loss parameters for the three benchmark models (MED, M1, and M2) for electron/positron propagation.

The electron/positron source term is given by

$$
q(\vec{x}, E)=\frac{1}{2 \eta}\langle\sigma v\rangle\left(\frac{\rho(\vec{x})}{m_{\mathrm{DM}}}\right)^{2} \frac{d N_{e}}{d E},
$$

where $\rho(\vec{x})$ is an assumed dark matter halo profile, $m_{\mathrm{DM}}$ is the dark matter mass, $\langle\sigma v\rangle$ is the average dark matter annihilation cross section in the galactic halo, and $d N_{e} / d E$ is the electron energy spectrum per dark matter annihilation. $\eta=1$ if dark matter is self-conjugate (e.g. a Majorana fermion), while $\eta=2$ if not (e.g. a Dirac fermion). We consider three spherically symmetric benchmark halo profiles (cored isothermal [31, NFW [32], and Einasto [33]) with $r_{\odot}=8.5 \mathrm{kpc}$ and $\rho_{\odot}=0.3 \mathrm{GeV} \mathrm{cm}^{-3}$ :

$$
\begin{aligned}
& \rho(r)_{\text {Isothermal }}=\rho_{\odot} \frac{1+\left(r_{\odot} / r_{c}\right)^{2}}{1+\left(r / r_{c}\right)^{2}}, \quad r_{c}=5 \mathrm{kpc}, \\
& \rho(r)_{\mathrm{NFW}}=\rho_{\odot} \frac{r_{\odot}}{r}\left(\frac{1+r_{\odot} / r_{c}}{1+r / r_{c}}\right)^{2}, \quad r_{c}=20 \mathrm{kpc}, \\
& \rho(r)_{\text {Einasto }}=\rho_{\odot} \exp \left\{-\frac{2}{\alpha}\left[\left(\frac{r}{r_{c}}\right)^{\alpha}-\left(\frac{r_{\odot}}{r_{c}}\right)^{\alpha}\right]\right\}, \quad \alpha=0.17, \quad r_{c}=20 \mathrm{kpc} .
\end{aligned}
$$

$N$-body simulations suggest that Einasto and NFW are more realistic profiles for $r \gtrsim 1 \mathrm{kpc}$. Within the inner region, however, there is considerable uncertainty, and we include the cored isothermal profile to explore the possibility of a less peaked distribution.

Once the source term is specified, Eq. (2) can be solved using the methods of [30, 34, and the electron/positron intensities (fluxes per energy per solid angle) at the earth due to dark matter annihilations are given by

$$
\frac{d \Phi_{e^{-} / e^{+}}^{(\mathrm{DM})}}{d E d \Omega}(E)=\frac{B_{e, \text { astro }}}{4 \pi} \psi_{e^{-} / e^{+}}\left(\vec{x}_{\odot}, E\right),
$$

where $\vec{x}_{\odot}$ is the location of the solar system and $B_{e \text {,astro }}$ is an astrophysical boost factor. Since the annihilation rate is proportional to the squared density of dark matter $\rho_{\mathrm{DM}}^{2}$, clumping of

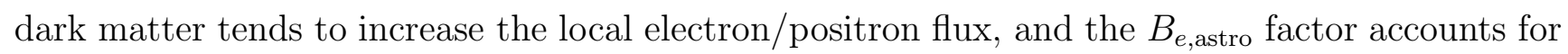
differences from the assumed smooth halo profile, as well as uncertainties in $\rho_{\odot}$. Of course, in the presence of clumpiness, the true electron/positron spectrum is also modified [35]. 
Galactic cosmic rays are a known source of background primary electrons. Background secondary electrons and positrons arise, e.g., from collisions of cosmic ray protons with interstellar gas. In principle, the spectra of background electrons/positrons are correlated with the diffusionloss parameters for electrons/positrons, but for simplicity we will use the parameterization of background primaries and secondaries from Ref. [36]:

$$
\begin{aligned}
\frac{d \Phi_{e^{-}}^{(\text {prim })}}{d E d \Omega} & =\frac{0.16 \varepsilon^{-1.1}}{1+11 \varepsilon^{0.9}+3.2 \varepsilon^{2.15}} \mathrm{GeV}^{-1} \mathrm{~cm}^{-2} \mathrm{~s}^{-1} \mathrm{sr}^{-1} \\
\frac{d \Phi_{e^{-}}^{(\mathrm{sec})}}{d E d \Omega} & =\frac{0.70 \varepsilon^{0.7}}{1+110 \varepsilon^{1.5}+600 \varepsilon^{2.9}+580 \varepsilon^{4.2}} \mathrm{GeV}^{-1} \mathrm{~cm}^{-2} \mathrm{~s}^{-1} \mathrm{sr}^{-1} \\
\frac{\Phi_{e^{+}}^{(\mathrm{sec})}}{d E d \Omega} & =\frac{4.5 \varepsilon^{0.7}}{1+650 \varepsilon^{2.3}+1500 \varepsilon^{4.2}} \mathrm{GeV}^{-1} \mathrm{~cm}^{-2} \mathrm{~s}^{-1} \mathrm{sr}^{-1}
\end{aligned}
$$

where again $\varepsilon=E / \mathrm{GeV}$. To treat background uncertainties, we will marginalize over the normalization and overall slope of the background in our analysis:

$$
\Phi_{e^{-}}^{(\text {back. fit })}=A_{-} \varepsilon^{P_{-}}\left(\Phi_{e^{-}}^{(\text {prim })}+\Phi_{e^{-}}^{(\text {sec })}\right), \quad \Phi_{e^{+}}^{(\text {back. fit })}=A_{+} \varepsilon^{P_{+}}\left(\Phi_{e^{+}}^{(\mathrm{sec})}\right)
$$

where we allow $0<A_{ \pm}<\infty,-0.05<P_{ \pm}<0.05$, as in [5].

In the limit that Eq. (1) holds, any given dark matter cascade topology has just two free parameters: the dark matter mass $m_{\mathrm{DM}}$ and the annihilation cross section $\langle\sigma v\rangle$. Following the literature, we normalize the cross section to the value that leads to the right relic thermal abundance $\langle\sigma v\rangle_{0} \simeq 3 \times 10^{-26} \eta \mathrm{cm}^{3} \mathrm{~s}^{-1}$ and express our results in terms of an effective boost factor

$$
B=B_{e, \text { astro }} \frac{\langle\sigma v\rangle}{\langle\sigma v\rangle_{0}},
$$

which includes both the deviation from the naive thermal freezeout cross section and dark matter clumping. Using only the statistical error bars, we perform a chi-squared fit of the derived electron/positron intensities to the PAMELA $e^{+} /\left(e^{+}+e^{-}\right)$and ATIC $e^{+}+e^{-}$data, treating $m_{\mathrm{DM}}$ and $B$ as free parameters. We use only $E \gtrsim 10 \mathrm{GeV}$ bins for the PAMELA data, as the lower energy bins are strongly affected by solar modulation effects (see the discussion in [1]).

The results of the fit for direct, 1-step, and 2-step annihilations are shown in the case of electron final states in Figure 2 and muon final states in Figure 3. (The results do not depend on whether the dark matter particle is self-conjugate or not.) Each plot corresponds to a definite cascade annihilation pattern with a definite halo profile, and shows $1 \sigma$ and $2 \sigma$ contours for three propagation models in the $m_{\mathrm{DM}^{-}} B$ plane. The best fit values for $m_{\mathrm{DM}}$ and $B$, as well as the $\chi^{2}$ values, are also given, where $\chi^{2}$ follows the chi-squared distribution with 22 degrees of freedom (7 PAMELA + 21 ATIC -6 fitting parameters: $\left.m_{\mathrm{DM}}, B, A_{ \pm}, P_{ \pm}\right)$. To evaluate goodness of fit, we also give $p$-values in Table 2, where we have chosen the propagation model giving smallest $\chi^{2}$ for each plot of Figures 2 and 3 . As we can see, the fits are reasonable for all the cases presented. 

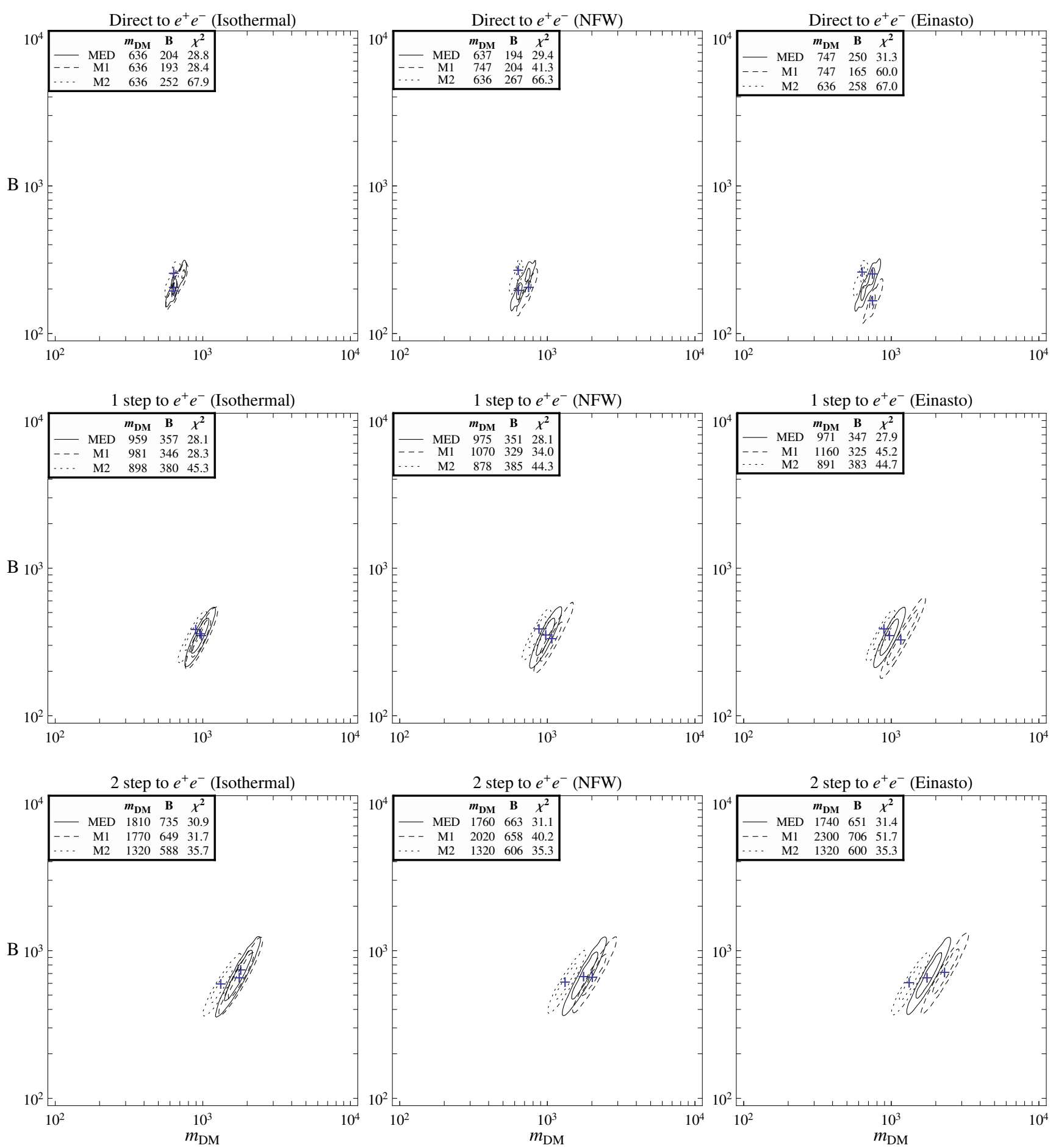

Figure 2: The best fit regions for the dark matter mass $m_{\mathrm{DM}}$ and boost factor $B$ in the cases of direct, 1-step, and 2-step annihilations into $e^{+} e^{-}$for different halo profiles and propagation models. The best fit values are indicated by the crosses, and the contours are for $1 \sigma$ and $2 \sigma$. 

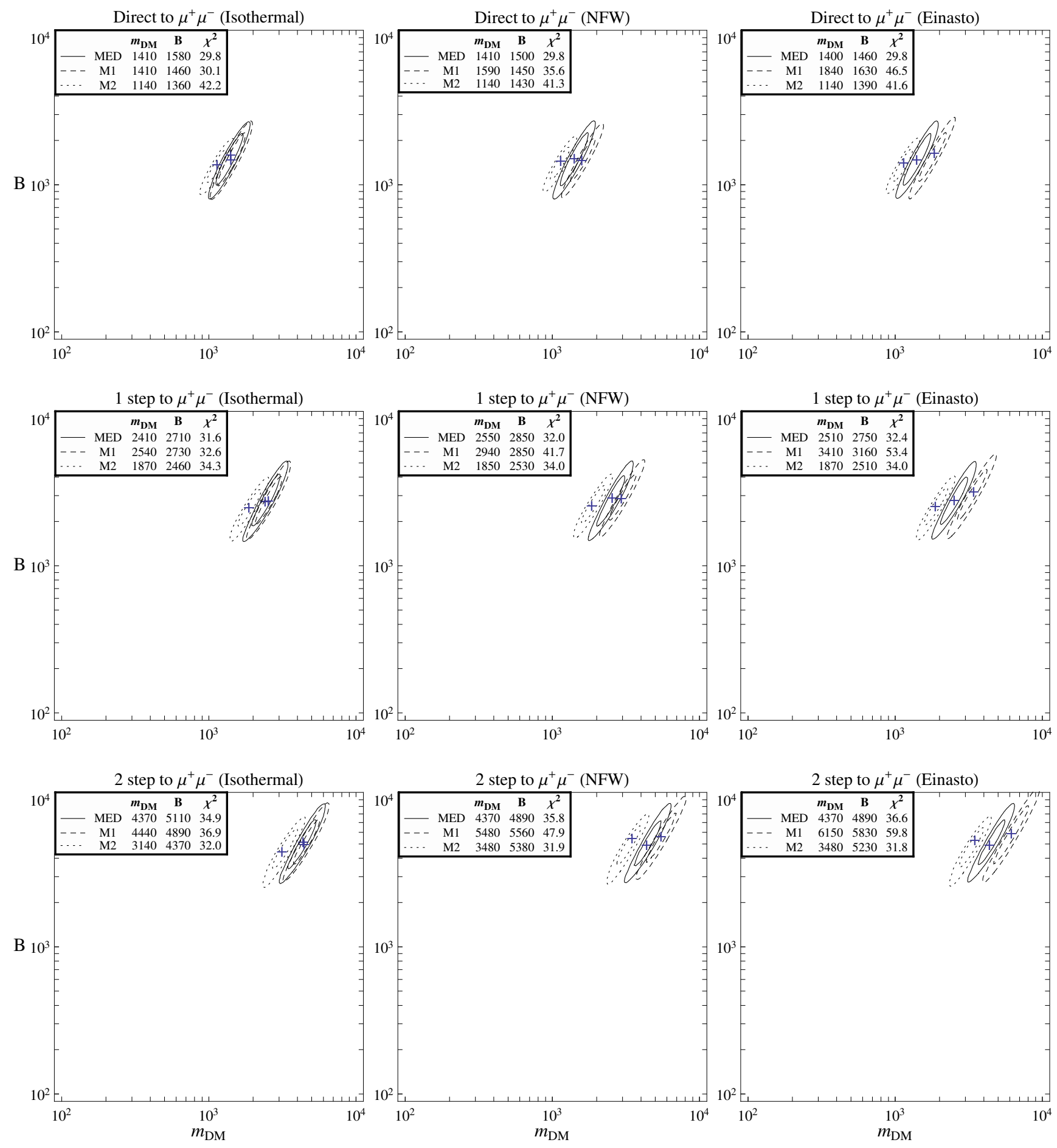

Figure 3: The same as Figure 2 but for annihilations into $\mu^{+} \mu^{-}$. 


\begin{tabular}{c|ccc|ccc} 
& Direct $e$ & 1 step $e$ & 2 step $e$ & Direct $\mu$ & 1 step $\mu$ & 2 step $\mu$ \\
\hline Isothermal & $16 \%$ & $17 \%$ & $9.8 \%$ & $12 \%$ & $8.5 \%$ & $7.7 \%$ \\
NFW & $13 \%$ & $17 \%$ & $9.4 \%$ & $12 \%$ & $7.7 \%$ & $7.9 \%$ \\
Einasto & $9.0 \%$ & $18 \%$ & $8.8 \%$ & $12 \%$ & $7.1 \%$ & $8.1 \%$
\end{tabular}

Table 2: The $p$-value for the best propagation model for each plot in Figures 2 and 3,
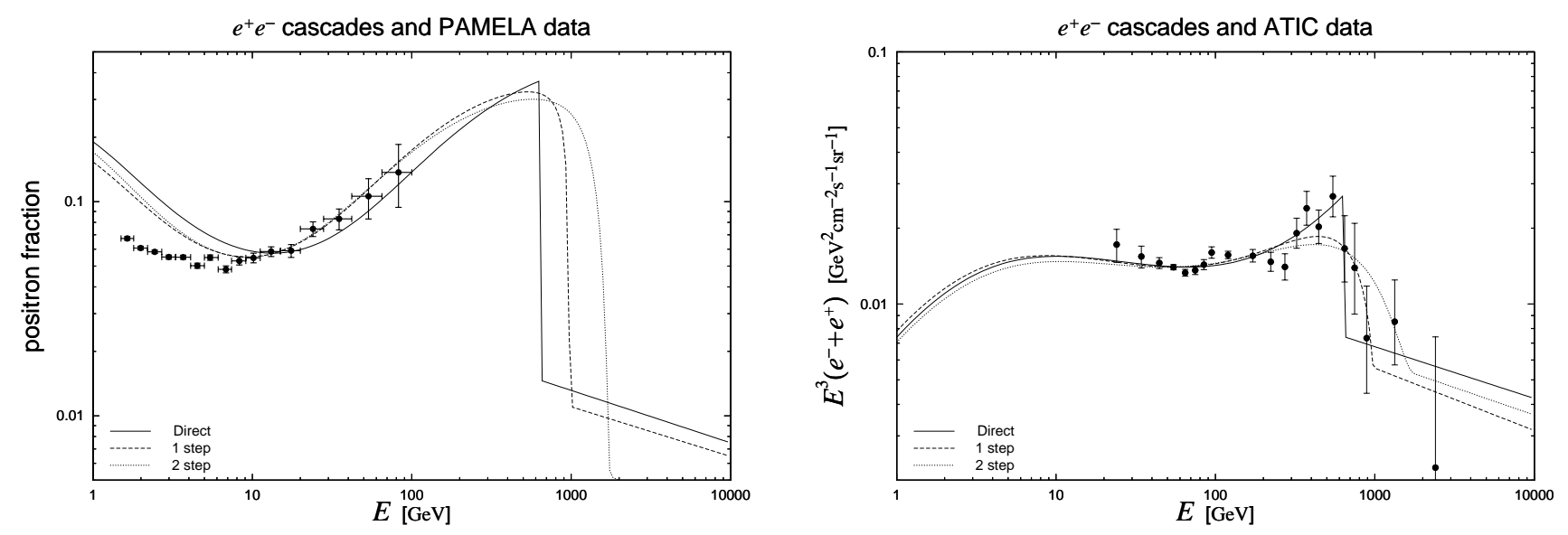

Figure 4: The predicted $e^{ \pm}$intensities compared to the PAMELA (left) and ATIC (right) data for direct (solid), 1-step (dashed), and 2-step (dotted) annihilations into electron final states. The NFW halo profile and the MED propagation model are chosen, and the $e^{ \pm}$backgrounds are marginalized as described in Eq. (11). Note that we fit the PAMELA data only for $E \gtrsim 10 \mathrm{GeV}$ because solar modulation effects are important at lower energies.
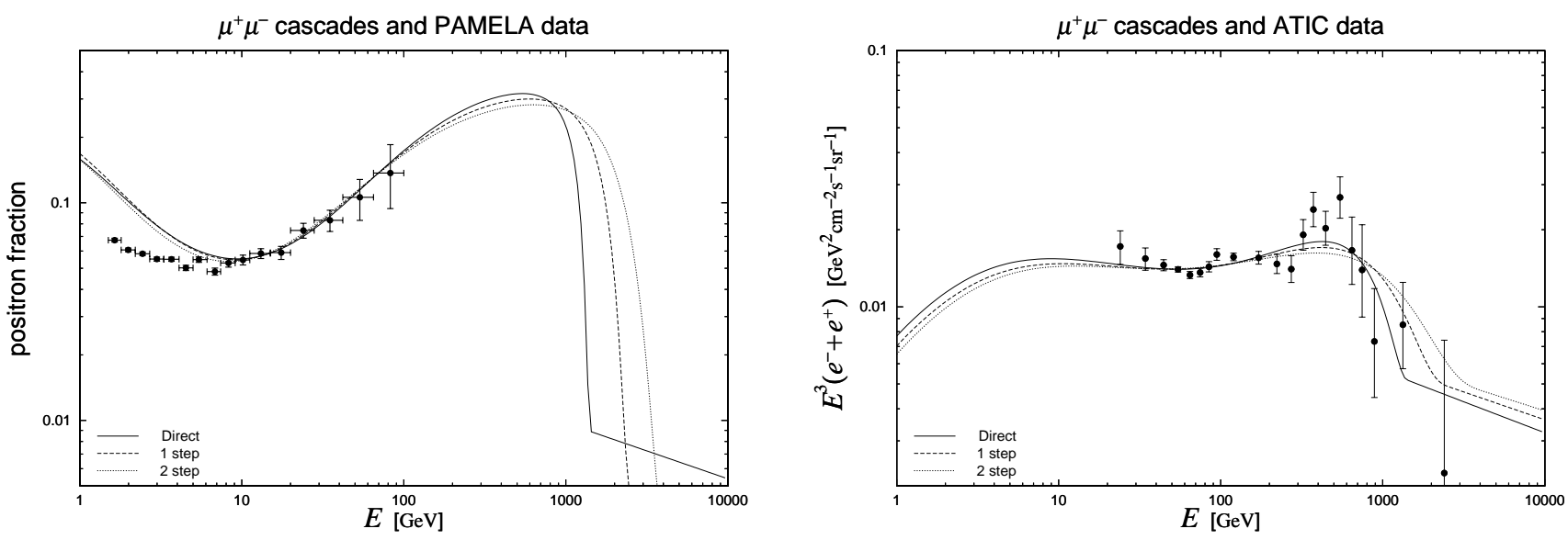

Figure 5: The same as Figure 4 but for annihilations into muon final states. 
In Figures 4 and 5, we show the comparisons of the $e^{ \pm}$intensities with the PAMELA and ATIC data in the cases of electron and muon final states. Solid, dashed, and dotted lines represent direct, 1-step, and 2-step annihilations, and we have chosen the NFW halo profile and the MED propagation model for illustrative purposes. We clearly see the trend that more steps in cascades lead to flatter $e^{ \pm}$spectra, but that the fits are good for all cases shown. Note that we have not optimized the propagation model here. Adjusting the propagation model can lead to a better fit in certain cases, especially the 2 -step annihilation into $\mu^{+} \mu^{-}$.

Summarizing the analysis of the $e^{ \pm}$data, we find:

- The PAMELA and ATIC data require the dark matter mass and boost factor in the region $m_{\mathrm{DM}}=O(\mathrm{TeV})$ and $B=O(1000)$, which is consistent with earlier analyses on the direct [5] and 1-step [8] cases. More steps in the cascade lead to larger values of $m_{\mathrm{DM}}$ and $B$, and roughly speaking, both $B$ and $m_{\mathrm{DM}}$ scale as $2^{n}$. The reason is that the peak location in the ATIC data sets the scale for the final state $e^{ \pm}$energy, and since the average $e^{ \pm}$energy over $m_{\mathrm{DM}}$ in an $n$-step cascade scales like $1 / 2^{n}, m_{\mathrm{DM}}$ must increase by $2^{n}$ to keep the peak location fixed. Similarly, the annihilation signal scales like $B N_{e} / m_{\mathrm{DM}}^{2}$ (assuming fixed $\rho_{\mathrm{DM}}$ ), where $N_{e}=2^{n+1}$ is the final state $e^{ \pm}$multiplicity. Thus, to keep the PAMELA/ATIC rate fixed, $B$ must scale like $2^{n}$.

- The fits do not become much worse by going to multiple steps, due to uncertainties in the highest energy ATIC data and uncertainties in the $e^{ \pm}$propagation model. In particular, 2-step annihilations still fit the data reasonably well for both electron and muon final states. The required boost factors are rather large in the case of muon final states: $B$ of a few thousand. Such large boost factors may come from both astrophysics, e.g. uncertainties in $\rho_{\odot}$ and nearby clumps of dark matter, and particle physics.

- Uncertainties in halo profiles and propagation models do not significantly affect the dark matter mass and the boost factor. Errors from these uncertainties are mostly of $O(10 \%)$ and at most a factor of 2 . The $2 \sigma$ ranges of the fits then determine $m_{\mathrm{DM}}$ and $B$ up to a factor of a few.

Recent measurements at H.E.S.S. [37] of the electron plus positron flux above $600 \mathrm{GeV}$ [38] are qualitatively consistent with the ATIC spectrum. Given the large systematic energy uncertainties and hadronic background, we do not use the H.E.S.S. data in our fits, although we remark that the observed steepening of the spectrum places some bounds on very long cascade decays and may disfavor spectra with a hard cutoff such as direct $e^{ \pm}$annihilation. 


\section{Gamma Ray Constraints}

When dark matter annihilates into charged leptons, there is a primary source of gamma rays coming from FSR. Various gamma ray telescopes have looked for excess gamma rays coming from the galactic center, and the null result of such searches puts bounds on dark matter annihilation into charged leptons. An additional effect that is beyond the scope of this paper is ICS, where electrons/positrons from dark matter annihilation lose energy by upscattering starlight photons into gamma rays. The rate of ICS photon production depends on the modeling of galactic starlight, and we here focus only on the bounds from FSR. For an early analysis of FSR in dark matter annihilation, see [39].

There is negligible energy loss as gamma rays propagate from the galactic center to the earth. The total power (flux per energy) on earth depends on the dark matter halo profile through

$$
\frac{d \Phi_{\gamma}}{d E}=\frac{B_{\gamma, \text { astro }}}{8 \pi \eta m_{\mathrm{DM}}^{2}}\langle\sigma v\rangle \bar{J} \Delta \Omega \frac{d N_{\gamma}}{d E},
$$

where $B_{\gamma \text {,astro }}$ is an astrophysical boost factor for photons that may differ from $B_{e \text {,astro, and }}$ $d N_{\gamma} / d E$ is the photon energy spectrum per dark matter annihilation. The energy spectrum from FSR is reviewed in Appendix A.3, and we also include the effect of radiative muon decays as described in Appendix A.4. $\Delta \Omega$ is the solid angle integration region, and $\bar{J}$ is the average line-of-sight-integrated squared dark matter density for a given halo model

$$
\bar{J}=\frac{1}{\Delta \Omega} \int_{\Delta \Omega} d \Omega \int_{\text {line-of-sight }} d s \rho(\vec{x})^{2} .
$$

The strongest bounds on FSR gamma rays come from atmospheric Cerenkov telescopes, but the way these experiments extract gamma ray signals affects the final dark matter annihilation bounds. To enable background subtraction, these telescopes operate either in on-off mode or wobble mode, meaning the effective $\bar{J}$ exposure is [40]

$$
\bar{J}_{\text {eff }}=\bar{J}_{\text {on-source }}-\bar{J}_{\text {off-source }} \text {. }
$$

By definition, $\bar{J}_{\text {eff }}$ is smaller than $\bar{J}_{\text {on-source }}$, and neglecting the $\bar{J}_{\text {off-source }}$ contribution gives bounds that are too aggressive. This means that one must know both the on- and off-source integration regions to derive a bound on the cross section. For shallow dark matter halo profiles, there can be large cancellations in the value of $\bar{J}_{\text {eff }}$, and in principle a stronger bound could be obtained using the raw unsubtracted data.

We set bounds on FSR using three H.E.S.S. gamma ray data sets. The first two are observations of the Galactic Center (GC) [20] and the Galactic Ridge (GR) [21]. Neither is ideal for dark matter observations because of the large contamination from gamma ray point sources and molecular gas, and in principle one should put bounds on a dark matter signal after subtracting 


\begin{tabular}{c|ccc|ccc} 
& GC (on) & GC (off) & GC (eff) & GR (on) & GR (off) & GR (eff) \\
\hline Isothermal & 10 & 10 & 0.028 & 10 & 10 & 0.019 \\
NFW & $1.1 \cdot 10^{4}$ & $3.6 \cdot 10^{2}$ & $1.1 \cdot 10^{4}$ & $1.8 \cdot 10^{3}$ & $4.3 \cdot 10^{2}$ & $1.4 \cdot 10^{3}$ \\
Einasto & $5.8 \cdot 10^{3}$ & $7.3 \cdot 10^{2}$ & $5.1 \cdot 10^{3}$ & $2.3 \cdot 10^{3}$ & $8.7 \cdot 10^{2}$ & $1.5 \cdot 10^{3}$
\end{tabular}

Table 3: $\bar{J}$ values for GC and GR gamma ray observations (on-source, off-source, and effective) in units of $\mathrm{GeV}^{2} \mathrm{~cm}^{-6} \mathrm{kpc}$.

both these foregrounds. Since such subtractions are not available, we derive conservative bounds by insisting that the dark matter signal does not exceed any of the H.E.S.S. data points by more than $2 \sigma$. For the GC and GR samples, values of $\bar{J}$ for the three dark matter halos in Eqs. (44, 5, 6) are shown in Table 3 for both the on-source and off-source regions.

The GC data set comes from the inner $0.1^{\circ}$ of the galaxy with a solid angle integration of $\Delta \Omega=1 \times 10^{-5}$, corresponding to the gamma ray source HESS J1745-290. This sample was taken in wobble mode, and the off-source region corresponds to a ring at a distance of $1.4^{\circ}$ from the GC. Apart from the off-source subtraction, no other corrections were made to the data, so the data points include both the HESS J1745-290 point source as well as any putative dark matter signal.

The GR data set comes from the region $|\ell|<0.8^{\circ},|b|<0.3^{\circ}$ in galactic coordinates, with foreground point sources HESS J1745-290 and G0.9+0.1 subtracted. The GR sample was taken in on-off mode, and the region $|\ell|<0.8^{\circ}, 0.8^{\circ}<|b|<1.5^{\circ}$ was used for background subtraction. In the GR data, H.E.S.S. finds that the gamma ray emissions are spatially correlated with molecular gas traced by CS emission lines, but a molecular gas foreground component is not subtracted from the data. The GR bounds are expected to strengthen if one were to subtract a molecular gas component.

H.E.S.S. looked more directly for dark matter annihilation in the Sagittarius dwarf spheroidal galaxy (Sgr dSph) [22]. Sgr dSph has negligible foregrounds and is thought to be dark matter dominated. A 95\% C.L. model-independent bound on the gamma ray flux was obtained for $E_{\gamma}>250 \mathrm{GeV}:$

$$
\Phi_{\gamma}\left(E_{\gamma}>250 \mathrm{GeV}\right)<3.6 \times 10^{-12} \mathrm{~cm}^{-2} \mathrm{~s}^{-1},
$$

with a solid angle integration of $\Delta \Omega=2 \times 10^{-5}$. Since the Sgr dSph data was taken in wobble mode and the contribution from the Sgr $\mathrm{dSph}$ halo is negligible in a $1.4^{\circ} \mathrm{ring}$, we use $\bar{J}_{\text {eff }}=\bar{J}_{\text {on-source }}$. The value of $\bar{J}_{\text {eff }}$ strongly depends on the halo profile of Sgr dSph. For example, an NFW profile, a large core profile, and a small core profile quoted in [15] lead to

$$
\bar{J}_{\mathrm{NFW}}^{\mathrm{Sgr} \mathrm{dSph}}=7.8 \times 10^{2}, \quad \bar{J}_{\text {Large Core }}^{\text {Sgr dSph }}=1.1 \times 10^{2}, \quad \bar{J}_{\text {Small Core }}^{\text {Sgr dSph }}=2.4 \times 10^{4},
$$

in units of $\mathrm{GeV}^{2} \mathrm{~cm}^{-6} \mathrm{kpc}$. To derive bounds, we consider NFW and large core profiles. 

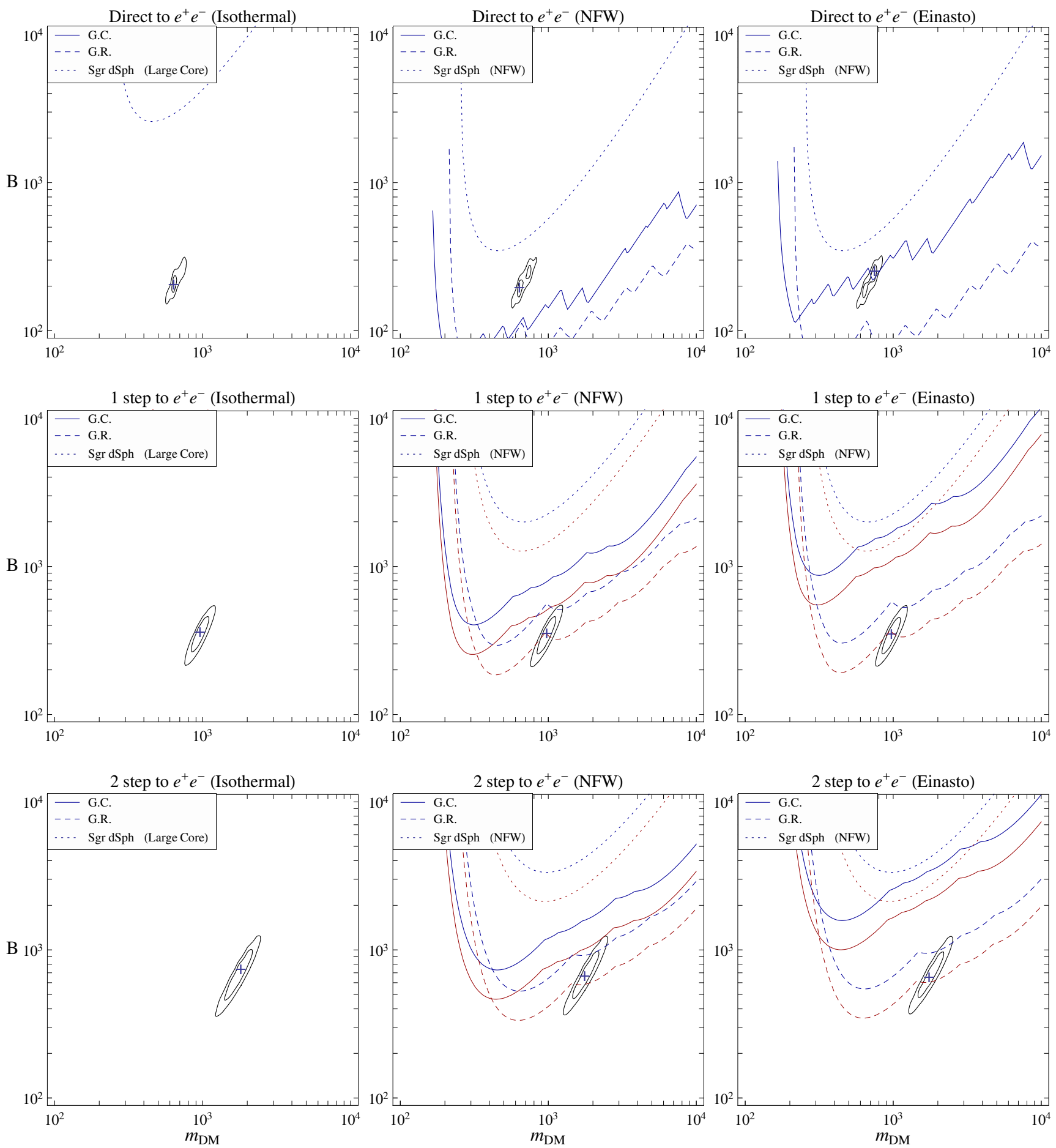

Figure 6: Constraints from gamma ray observations, GC (solid), GR (dashed), and Sgr dSph (dotted), in the $m_{\mathrm{DM}^{-}} B$ plane for direct, 1-step, and 2-step annihilations into electron final states. All the constraints, as well as the best fit region for PAMELA/ATIC (MED propagation), are plotted assuming $B_{e \text {,astro }}=B_{\gamma, \text { astro }}$. For cascade annihilations, each of the GC, GR, and Sgr dSph constraints consist of two curves, with the upper (blue) and lower (red) curves corresponding to $m_{1}=100 \mathrm{MeV}$ and $1 \mathrm{GeV}$, respectively. Note that the constraint lines in the cored isothermal case are above the plot region, and that the halo profiles for Sgr dSph are given in the legends. 

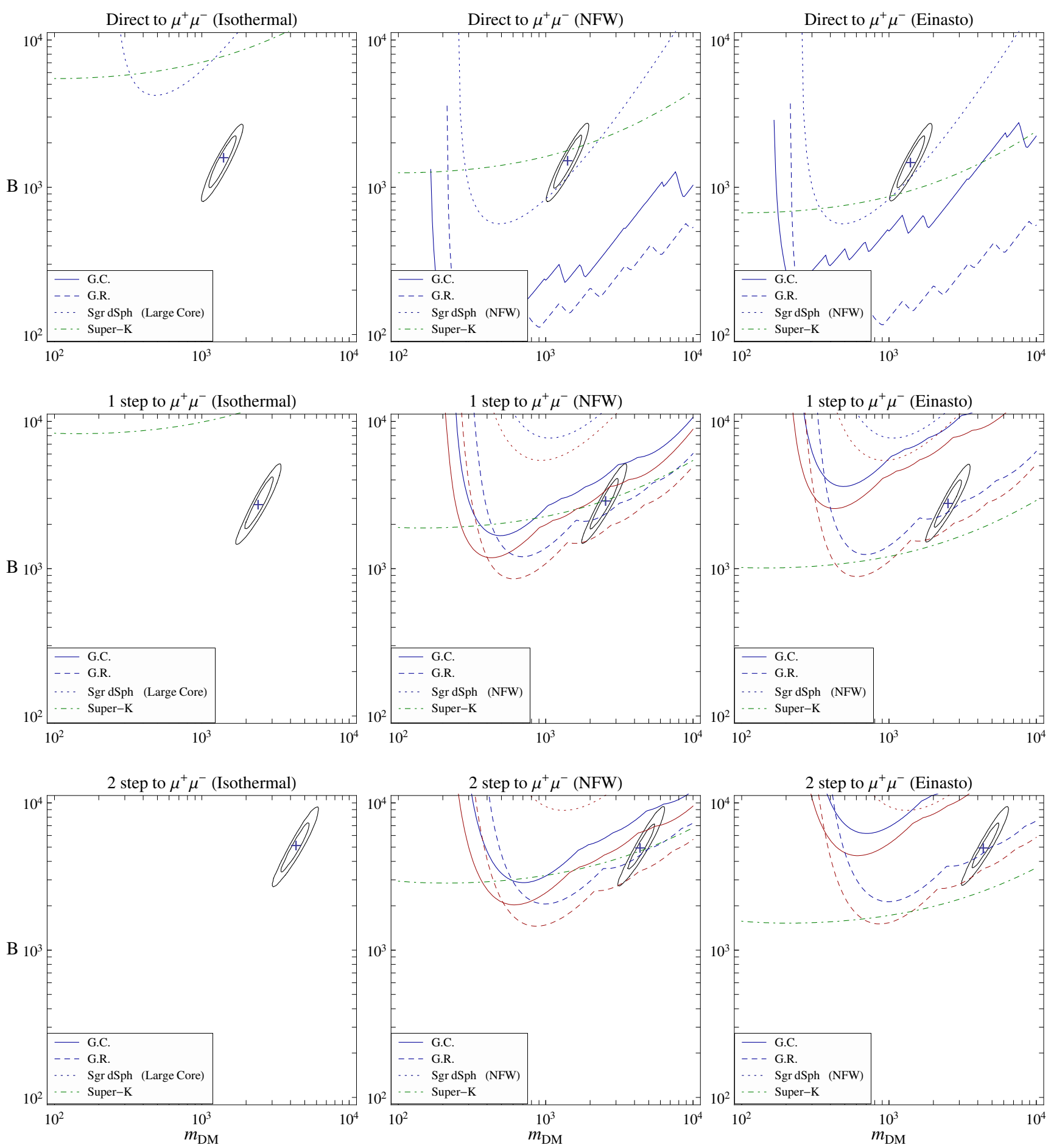

Figure 7: The same as Figure 6 but for muon final states. Also included are constraints from neutrino observations (dot-dashed) assuming $B_{\nu, \text { astro }}=B_{e, \text { astro. }}$ For cascade annihilations, the upper (blue) and lower (red) curves now correspond to $m_{1}=600 \mathrm{MeV}$ and $1 \mathrm{GeV}$, respectively. 
In Figures 6 and 7, we show the resulting constraints from the GC, GR, and Sgr dSph gamma ray observations in the $m_{\mathrm{DM}^{-}} B$ plane. We also superimpose the $1 \sigma$ and $2 \sigma$ contours reproducing the PAMELA/ATIC data for the MED propagation model; see Figures 2 and 3 . In order to plot the Sgr dSph and Milky Way bounds on the same plane, we associate the Sgr dSph small core profile with the Milky Way cored isothermal profile, and the Sgr dSph NFW profile with the Milky Way NFW and Einasto profiles. The ragged lines in the GC and GR constraints come from the binning of the H.E.S.S. data.

We note that here we have drawn the gamma ray constraints and $e^{ \pm}$contours assuming a

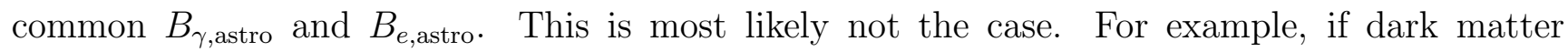
clumping decreases toward the galactic center, then the gamma ray flux will decrease compared to the local positron flux. If the astrophysical boost factor for $e^{ \pm}$is larger than that for $\gamma$, then the gamma ray constraints become weaker by a factor of $B_{e \text {,astro }} / B_{\gamma \text {,astro }}$ compared to those shown in Figures 6 and 7.

The bounds from Sgr dSph can be modified if there are nonperturbative enhancements to the dark matter annihilation cross section. Since the velocity dispersion of dark matter in Sgr dSph is $v_{\mathrm{Sgr}} \sim 10 \mathrm{~km} / \mathrm{s}$ [41], as opposed to $v_{\mathrm{MW}} \sim 200 \mathrm{~km} / \mathrm{s}$ in the Milky Way, the boost factor relevant for Sgr dSph may be larger than that for electrons/positrons if part of the boost factor arises from the Sommerfeld or bound state enhancement. This would make the Sgr dSph constraint stronger than what is naively read from Figures 6 and 7.

Summarizing the analysis of the gamma ray constraints, we find:

- For FSR gamma rays from the galactic center region, the GR data gives somewhat stronger constraints than the GC data. For direct annihilation, this disfavors the NFW and Einasto profiles, as also seen in Refs. [14, 15]. The Sgr dSph data is not constraining unless we were to take a highly peaked halo such as the small core profile.

- The constraints from FSR photons are weaker in the cascade annihilation case than in the direct annihilation case. This is because cascade annihilations give smaller photon yield at high energies, as discussed in Appendix A.3. The smaller the $\phi_{1}$ mass is, the weaker the constraints become. The constraints, however, do not become weaker by increasing the number of steps, as can be seen by comparing the best fit region for the $e^{ \pm}$data with the gamma ray constraints. This is because the reduction in the photon yield depends only on the $\phi_{1}$ mass and not on the number of cascade steps, and the softening of the gamma ray spectra is compensated by the increase in the best fit dark matter mass and boost factor.

- The constraints are very weak for shallow halo profiles such as the cored isothermal profile for the Milky Way in Eq. (4). This is particularly true for the GC and GR data because the background subtraction due to on-off or wobble mode operation also subtracts (most of) the signals from dark matter annihilations. The constraints from these data, therefore, are 
rather weak as long as the halo profile is relatively flat within about $100 \mathrm{pc}$ of the galactic center. A better bound may be obtained if we could use the unsubtracted data.

While we have focused only on FSR photons in our analysis, we wish to make a few comments about ICS, the WMAP Haze, and radio bounds. In the context of dark matter, the WMAP Haze arises because electrons from dark matter annihilation emit synchrotron radiation in the galactic magnetic fields. The total synchrotron power - and hence the size of the predicted WMAP Haze signal-depends on whether these electrons can lose energy via non-synchrotron channels. Ref. [8] found that in order to be consistent with the large boost factors necessary to explain PAMELA/ATIC, one had to assume a larger rate for ICS compared to earlier Haze analyses [11]. Given the uncertainty in galactic starlight and the dark matter halo profile, it is consistent to conservatively ignore potential bounds from ICS, but since the WMAP Haze is one of the motivations for considering dark matter annihilation, strictly speaking one should verify that the assumed electron energy loss mechanisms can yield the WMAP Haze while satisfying ICS photon bounds.

That said, we do not expect much variation in the ICS bounds between direct and cascade annihilation scenarios. ICS is calculated from a steady state configuration of charged particles, so to the extent that the PAMELA/ATIC data already normalizes the steady state electron/positron densities, the ICS yield should be similar regardless of the annihilation scenario. Therefore, the recent analysis of Ref. [8] should be representative of generic multi-step cascade annihilation scenarios. This is similar in spirit to the WMAP Haze, in that the Haze requires a source of charged particles to generate the synchrotron signal, but the precise particle energy distribution has only a secondary effect.

Finally, there has also been recent suggestions [15, 16] of a possible tension between a dark matter annihilation interpretation of the WMAP Haze and bounds from $408 \mathrm{MHz}$ radio observations in the inner $4^{\prime \prime}$ of the Milky Way [42. Such bounds assume that the steep halo profiles necessary to generate the Haze at a latitudinal distance between $5^{\circ}$ and $30^{\circ}$ can be extrapolated to sub-parsec distances away from the galactic center. There are a number of reasons to distrust such an extreme extrapolation of the dark matter halo, including possible effects of baryons [43] and hierarchical mergers [44] to soften cuspy behavior. At minimum, $N$-body simulations [45] do not have resolution to such small scales. Therefore, we find no reason to disfavor a dark matter annihilation scenario on the basis of the $408 \mathrm{MHz}$ radio bound. Note that the analysis of Ref. [46] using an all-sky radio model [47] finds only relatively mild synchrotron constraints on TeV-scale dark matter after masking the inner $15^{\circ} \times 15^{\circ}$ of the galaxy. 


\section{$5 \quad$ Neutrino Constraints}

When dark matter annihilates into muons, there is an irreducible source of neutrinos. Neutrinos produced in the galactic center oscillate as they travel towards earth, and if they are muon-type neutrinos when they collide with rock in the earth's crust, they can create an upward-going flux of muons. These muons could be observed by water Cerenkov detectors, and the absence of such observations puts bounds on the dark matter annihilation rate into muons. For muon cascades, there is no high energy neutrino source from dark matter that accretes in the sun and earth, because the muons from dark matter annihilation are stopped before they decay [48].

Since neutrinos have negligible energy losses as they traverse the galaxy, the muon-neutrino flux incident on earth is

$$
\frac{d \Phi_{\nu_{\mu}}}{d E_{\nu}}=\sum_{i} P_{\nu_{i} \nu_{\mu}} \frac{B_{\nu, \text { astro }}}{8 \pi \eta m_{\mathrm{DM}}^{2}}\langle\sigma v\rangle \bar{J} \Delta \Omega \frac{d N_{\nu_{i}}}{d E},
$$

where $i$ runs over the neutrino flavors, $B_{\nu \text {,astro }}$ is the astrophysical boost factor for the neutrino signal which could differ from $B_{e, \text { astro, }}$ and $P_{\nu_{i} \nu_{\mu}}$ is the probability that $\nu_{i}$ has oscillated into $\nu_{\mu}[49]$ :

$$
P_{\nu_{\mu} \nu_{\mu}}=0.39, \quad P_{\nu_{e} \nu_{\mu}}=0.21
$$

There is an analogous formula for $\bar{\nu}_{\mu}$, but in dark matter annihilations the $\nu_{\mu}$ and $\bar{\nu}_{\mu}$ fluxes are equal. The primary neutrino spectra are given in Appendix A.2.

We now calculate the resulting upward-going muon flux following the analysis of Ref. [50]. An incident neutrino of energy $E_{\nu}$ creates muons of energy $E_{\mu}$ according to the neutrino-nucleon scattering cross sections $\sigma_{\nu N \rightarrow \mu X}$. For the propagation of created muons, we use an approximate energy loss parameterization

$$
\frac{d E}{d L}=\rho_{\text {mat }}(-\alpha-\beta E),
$$

with "standard rock" values $\alpha=2 \times 10^{-6} \mathrm{TeV} \mathrm{cm} / \mathrm{g}$ and $\beta=4 \times 10^{-6} \mathrm{~cm}^{2} / \mathrm{g} ; \rho_{\text {mat }}$ will cancel in the final muon flux expression. In this approximation, a muon of starting energy $E_{\mu}$ can travel a distance

$$
L\left(E_{\mu}, E_{\text {thres. }}\right)=\frac{1}{\rho_{\text {mat }} \beta} \ln \left(\frac{\alpha+\beta E_{\mu}}{\alpha+\beta E_{\text {thres. }}}\right)
$$

before its energy drops below the muon detection threshold $E_{\text {thres. }}$.

For a given $d \Phi_{\nu_{\mu}} / d E_{\nu}$, the observed muon flux is

$$
\Phi_{\mu}=\int_{E_{\text {thres. }}}^{m_{\mathrm{DM}}} d E_{\mu} \int_{E_{\mu}}^{m_{\mathrm{DM}}} d E_{\nu} n_{N}\left(\frac{d \Phi_{\nu_{\mu}}}{d E_{\nu}} \frac{d \sigma_{\nu_{\mu} N \rightarrow \mu^{-} X}}{d E_{\mu}}+\frac{d \Phi_{\bar{\nu}_{\mu}}}{d E_{\nu}} \frac{d \sigma_{\bar{\nu}_{\mu} N \rightarrow \mu^{+} X}}{d E_{\mu}}\right) L\left(E_{\mu}, E_{\text {thres. }}\right),
$$

where $n_{N}=\rho_{\text {mat }} / m_{N}$ is the nucleon number density in the earth's crust, and $N$ refers to an average nucleon. We calculate the neutrino-nucleon scattering cross sections assuming an equal 


\begin{tabular}{c|ccccccc} 
& $3^{\circ}$ & $5^{\circ}$ & $10^{\circ}$ & $15^{\circ}$ & $20^{\circ}$ & $25^{\circ}$ & $30^{\circ}$ \\
\hline Isothermal & 10 & 10 & 9.7 & 9.0 & 8.2 & 7.4 & 6.6 \\
NFW & 340 & 190 & 84 & 51 & 35 & 26 & 20 \\
Einasto & 640 & 370 & 160 & 91 & 60 & 43 & 32 \\
\hline Super-K (95\% C.L.) & 2.70 & 4.82 & 6.43 & 10.6 & 11.2 & 17.6 & 19.5
\end{tabular}

Table 4: $\bar{J}$ values for neutrino observations in units of $\mathrm{GeV}^{2} \mathrm{~cm}^{-6} \mathrm{kpc}$, and Super-K 95\% C.L. flux limits in units of $10^{-15} \mathrm{~cm}^{-2} \mathrm{~s}^{-1}$.

fraction of protons and neutrons in rock, using CTEQ5M parton distribution functions [51] to include the effect of sea quarks, and retaining the full $W$ boson propagator in the cross section.

Super-K [52] placed 90\% confidence bounds on the upward-going muon flux [25] in various cone sizes ranging from $3^{\circ}$ to $30^{\circ}$ around the galactic center, with $E_{\text {thres. }}=1.6 \mathrm{GeV}$. To be more conservative, we consider 95\% confidence bounds [53], as shown in Table 4. The relevant values of $\bar{J}$ for the three dark matter halos in Eqs. (4, 5, 6) are also shown in Table 4. To derive a bound on the annihilation rate, we insist that the predicted flux does not exceed the $95 \%$ confidence bound for any of the Super-K cone sizes. The neutrino constraints for muon cascade scenarios appear in Figure 7, assuming a common boost factor for the electron, gamma ray, and neutrino signals.

As observed in Ref. [48, for sufficiently small dark matter masses, the observed muon flux is nearly independent of the dark matter mass. The reason is that both the neutrino-nucleon scattering cross section and the muon range scale like energy, but the dark matter number density squared (and hence the annihilation signal) scales like $1 / m_{\mathrm{DM}}^{2}$, so the final observed flux is simply related to the normalized second-moment of the neutrino energy spectrum. Hence the exclusion limit for the neutrino boost factor is approximately flat in $m_{\mathrm{DM}}$ for small enough dark matter masses. As the dark matter mass increases, the average neutrino gets harder, and the neutrinonucleon cross section grows less steeply because of the $W$ boson propagator. In addition, the energy-dependent term in $d E / d L$ begins to take effect, relaxing the neutrino bounds for high dark matter masses.

Summarizing the analysis of the neutrino constraints, we find:

- Since the neutrinos are softer in cascade annihilations, the bounds on the boost factor are weaker than for direct annihilation. However, the electrons are also softer in cascade annihilations, so the PAMELA/ATIC best fit mass and boost factor rise. Put together, the neutrino tension increases marginally as the number of cascade steps increase. For direct annihilation, our results agree qualitatively with [17, 18].

- Super-K considered solid angles as large as $30^{\circ}$, so a large fraction $(\simeq 30 \%$ to $\simeq 70 \%)$ of the total dark matter annihilation signal is contained within the observed region. While 


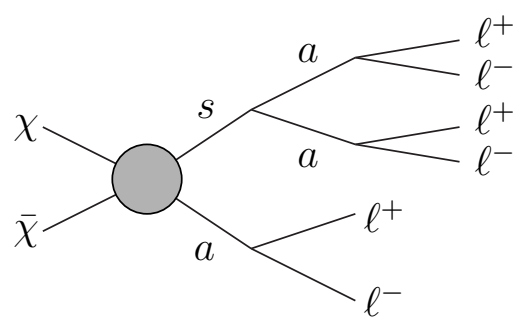

Figure 8: In the axion portal, fermionic dark matter annihilates dominantly into a scalar $s$ and a pseudoscalar "axion" $a$. The scalar then decays as $s \rightarrow a a$, and the axion decays as $a \rightarrow \ell^{+} \ell^{-}$. In the minimal axion portal, the axion dominantly decays into muons, but in the leptonic axion portal it can dominantly decay into electrons. These models are partway between a 1-step and a 2-step cascade annihilation scenario.

the Einasto profile is less peaked than NFW toward the galactic center, the Einasto bound happens to be stronger because of the large integration region. For the NFW and Einasto profiles, the dominant bounds come from the $10^{\circ}$ cone, while for the cored isothermal profile they come from the $30^{\circ}$ cone.

- Since the gross structure of the dark matter halo is better understood than the halo density at the galactic center, the Super-K neutrino constraints are in some sense more robust than the H.E.S.S. gamma ray constraints. As in the case of gamma rays, the bounds are rather weak for the cored isothermal profile. They are, however, significantly stronger in the more realistic NFW and Einasto profiles. For cascade decays, the neutrino constraints are comparable to or stronger than the FSR gamma ray constraints, and highlight the tension in muon cascade scenarios. As with the gamma ray bounds, however, differences between the astrophysical boost factors $B_{e \text {,astro }}$ and $B_{\nu \text {,astro }}$ could alleviate the tension.

\section{The Axion Portal}

One well-motivated example of a cascade annihilation scenario is the axion portal [7]. In this scenario, dark matter is a $\mathrm{TeV}$-scale particle that obtains a mass from spontaneous symmetry breaking. The spontaneous breaking of $U(1)_{X}$ yields a pseudoscalar "axion" $a$ and a scalar "Higgs" $s$, and for fermionic dark matter the dominant annihilation channel is

$$
\chi \bar{\chi} \rightarrow s a
$$

$(\chi \chi \rightarrow s a$ if $\chi$ is a Majorana fermion). The scalar $s$ dominantly decays as $s \rightarrow a a$, and if standard model leptons carry axial $U(1)_{X}$ charges, then $a \rightarrow \ell^{+} \ell^{-}$. Since $a$ is a pseudoscalar, helicity suppression implies $a$ will decay into the heaviest kinematically allowed lepton, which we assume 


\begin{tabular}{c|c|c} 
& Minimal Axion Portal & Leptonic Axion Portal \\
\hline Isothermal & $7.9 \%$ & $9.2 \%$ \\
NFW & $7.9 \%$ & $9.2 \%$ \\
Einasto & $8.3 \%$ & $8.8 \%$
\end{tabular}

Table 5: The $p$-values for the best propagation model for each plot in Figure 9 ,

is either an electron or muon. An exchange of $s$ can also provide the necessary enhancement of the annihilation cross section through the Sommerfeld and/or bound state effect. As shown in Figure 8, the axion portal effectively gives a one-and-a-half step cascade annihilation in the language used here.

The simplest model for the axion portal - the minimal axion portal - is obtained if we identify $U(1)_{X}$ with a Peccei-Quinn symmetry rotating two Higgs doublets. In this case, a has large hadronic couplings, and there are strong constraints on the axion mass from beam dump experiments and rare meson decays. Ref. [7] found that the preferred axion mass range was $360 \mathrm{MeV} \lesssim m_{a} \lesssim 800 \mathrm{MeV}$, in which case $a$ preferentially decays into muons. In the analysis here, we also consider a variant of the axion portal - the leptonic axion portal - where only leptons are charged under $U(1)_{X}$. This possibility was mentioned in [7, and is described in more detail in Appendix B. Dark matter in this model annihilates through the axion $a_{\ell}$ associated with the leptonic symmetry, which does not have a coupling to hadrons. The absence of hadronic couplings allows the parameter range $2 m_{e}<m_{a_{\ell}}<2 m_{\mu}$, so $a_{\ell}$ can preferentially decay into electrons. Note that the nonperturbative enhancement of the halo cross section is caused by the exchange of another scalar $s_{\ell}$ and not by the axion $a_{\ell}$, so the bound of Ref. [54] does not exclude $a_{\ell}$ masses smaller than $\approx 100 \mathrm{MeV}$.

In Figure 9, we show the best fit values for $m_{\mathrm{DM}}$ and $B$ for the PAMELA/ATIC data for the three different diffusion models and three different halo profiles. In Figure 10, we compare the best fit regions to FSR and neutrino constraints. Here we assume that $B$ is common for electron, gamma ray, and neutrino signals, so that the same qualifications as Figures 6 and 7 apply. To account for the fact that smaller masses for $a_{\ell}$ are allowed, we are considering smaller values of $m_{a_{\ell}}$ in Figure 10 than those of $m_{1}$ in Figure 6. For completeness, we also show the best fit spectra to the PAMELA and ATIC data in Figure 11, and the $p$-values of the fit in Table 5. As expected, the best fit values and qualitative features of the plots are partway between a 1-step and a 2-step cascade scenario.

Depending on the axion mass, there are also potential gamma ray constraints from rare $a \rightarrow \gamma \gamma$ and $a \rightarrow \pi^{+} \pi^{-} \pi^{0}$ decay modes, and we can place bounds on the branching fractions to these modes. The $a \rightarrow \gamma \gamma$ mode is potentially dangerous for the entire axion mass range, but the $a \rightarrow \pi^{+} \pi^{-} \pi^{0}$ mode only when $m_{a}>3 m_{\pi}$. The gamma ray spectra for these rare decays are given 

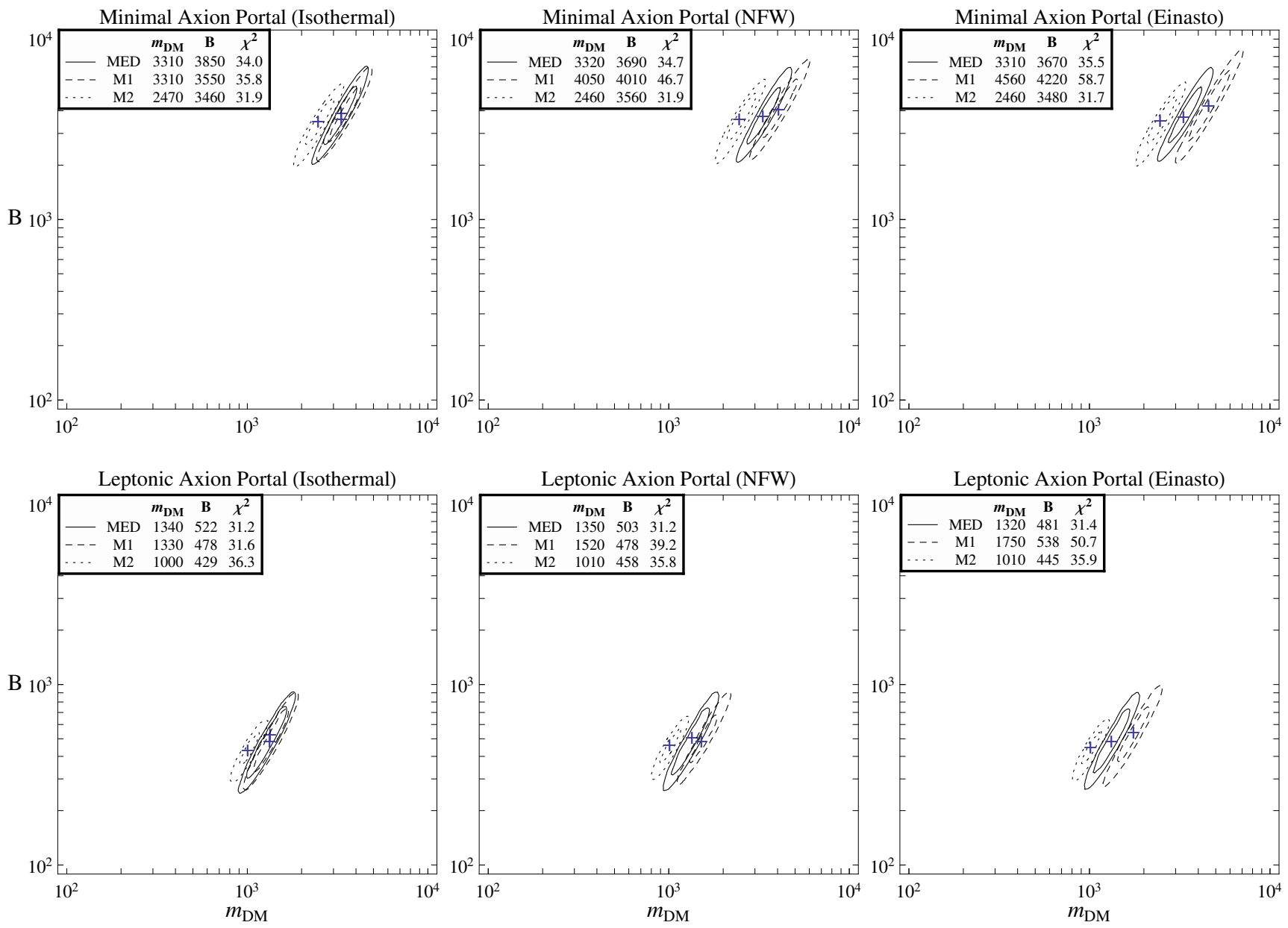

Figure 9: The best fit regions for the dark matter mass $m_{\mathrm{DM}}$ and boost factor $B$ in the minimal axion portal (top row, $a \rightarrow \mu^{+} \mu^{-}$) and leptonic axion portal (bottom row, $a_{\ell} \rightarrow e^{+} e^{-}$) for different halo profiles and propagation models. The best fit values are indicated by the crosses, and the contours are for $1 \sigma$ and $2 \sigma$. 

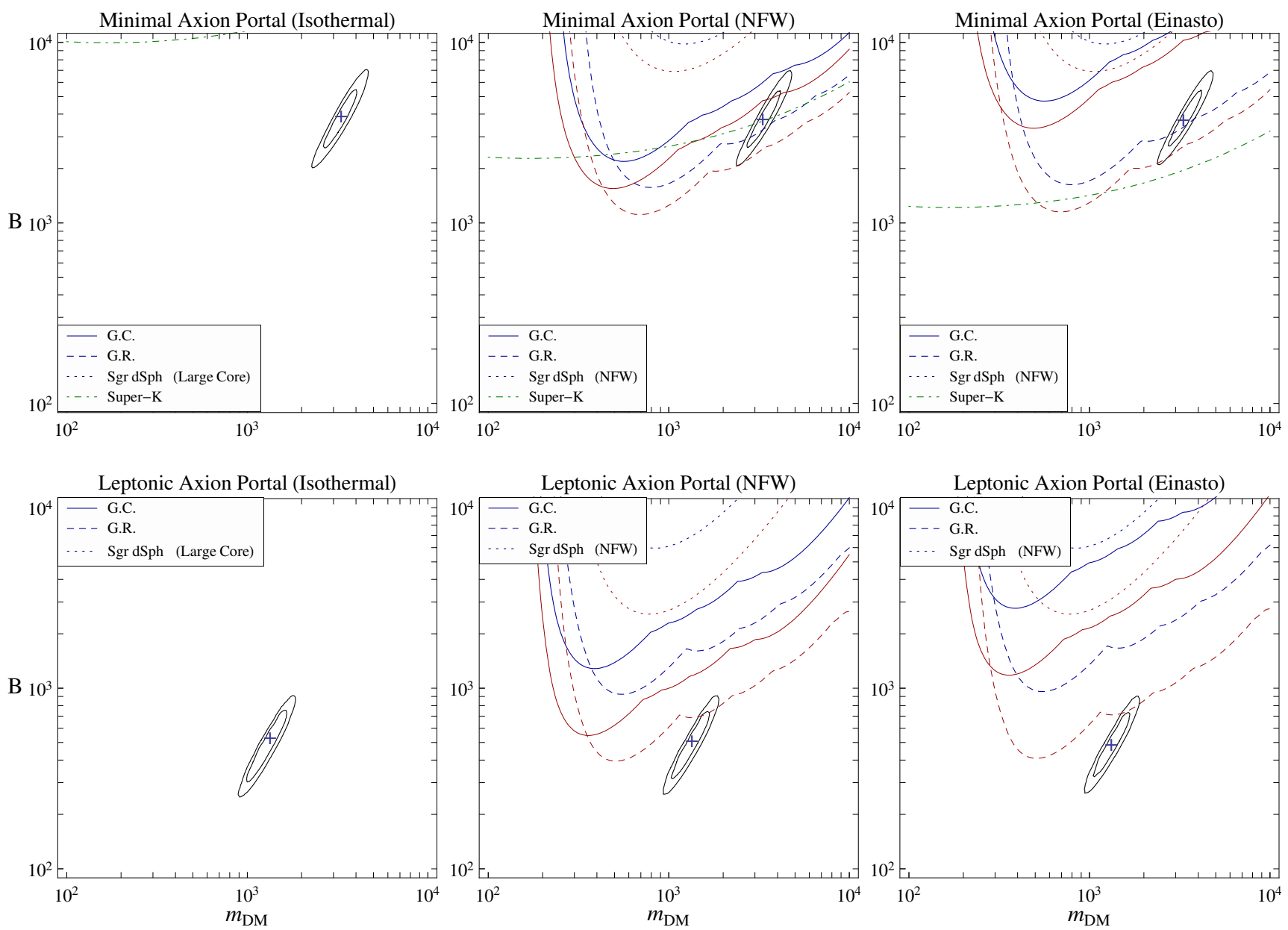

Figure 10: Constraints from gamma ray, GC (solid), GR (dashed), and Sgr dSph (dotted), and neutrino (dot-dashed) observations in the $m_{\mathrm{DM}^{-}}-B$ plane in the minimal axion portal (top row, $a \rightarrow \mu^{+} \mu^{-}$) and leptonic axion portal (bottom row, $a_{\ell} \rightarrow e^{+} e^{-}$). All the constraints, as well as the best fit region for PAMELA/ATIC (MED propagation), are plotted assuming that $B$ is common. Each of the GC, GR, and Sgr dSph constraints consist of two curves. For the minimal axion portal, the upper (blue) curve is $m_{a}=600 \mathrm{MeV}$ and the lower (red) curve is $m_{a}=1 \mathrm{GeV}$. For the leptonic axion portal, the upper (blue) curve is $m_{a_{\ell}}=10 \mathrm{MeV}$ and the lower (red) curve is $m_{a_{\ell}}=100 \mathrm{MeV}$, which differs from the choice in Figure 6. Note that the constraint lines in the cored isothermal case are above the plot region, and that the halo profiles for Sgr dSph are given in the legends. 

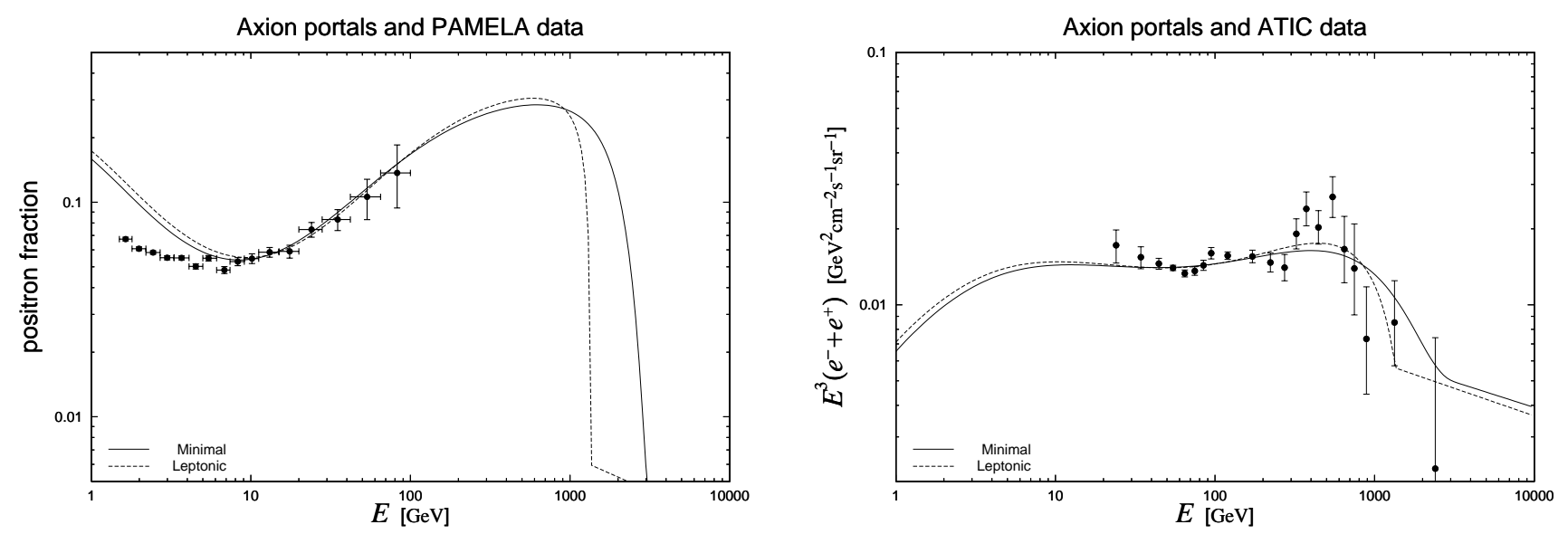

Figure 11: The predicted $e^{ \pm}$intensities compared to the PAMELA (left) and ATIC (right) data for the minimal axion portal (solid, $a \rightarrow \mu^{+} \mu^{-}$) and leptonic axion portal (dashed, $a_{\ell} \rightarrow e^{+} e^{-}$). The NFW halo profile and the MED propagation model are chosen, and the $e^{ \pm}$backgrounds are marginalized as described in Eq. (11). Note that we fit the PAMELA data only for $E \gtrsim 10 \mathrm{GeV}$ because solar modulation effects are important at lower energies.

in Appendix A.5. For each halo profile and for the three H.E.S.S. data sets, we turn off FSR and find the values of the branching ratios where the H.E.S.S. bound is saturated for the best fit values of $m_{\mathrm{DM}}$ and $B$ and the best propagation model (assuming $B$ is common for $e^{ \pm}$and gamma rays).

The branching ratio bounds are shown in Tables 6 and 7. The constraints from Sgr dSph match the estimates in [7, but the GC and GR data imply a factor of 5-10 stronger bound. In the minimal axion portal, $\operatorname{Br}(a \rightarrow \gamma \gamma) \sim 10^{-5}-10^{-4}$, which is safe from the bounds. There is somewhat more tension for $a \rightarrow \pi^{+} \pi^{-} \pi^{0}$ where the estimated branching fraction was $\sim 10^{-2}$. Note, however, that the constraints from the GC and GR data are weak for any halo profile relatively flat within about $100 \mathrm{pc}$ of the galactic center. In particular, there is no meaningful branching ratio constraint for the cored isothermal profile in Eq. (4).

Gamma ray and radio bounds on the axion portal were also considered in Ref. [16], where potential $s \rightarrow b \bar{b}$ and $s \rightarrow \tau^{+} \tau^{-}$decay modes were included. Since these additional $s$ decay modes depend on details of the model, we do not consider them here. For FSR gamma rays, our results and conclusions agree qualitatively with [16]. As mentioned already, we do not consider $408 \mathrm{MHz}$ radio observations to place any meaningful constraint on the axion portal.

\section{Conclusions}

The possibility of indirect detection of dark matter has been considered for over 25 years [55], but the annihilation rates expected from WIMP thermal relics are typically too small to give 


\begin{tabular}{c|ccc}
$\operatorname{Br}(a \rightarrow \gamma \gamma)$ & GC & GR & Sgr dSph \\
\hline NFW Best & $2.2 \cdot 10^{-3}$ & $1.1 \cdot 10^{-3}$ & $2.5 \cdot 10^{-2}$ \\
Einasto Best & $4.8 \cdot 10^{-3}$ & $1.2 \cdot 10^{-3}$ & - \\
$\operatorname{Br}\left(a \rightarrow \pi^{+} \pi^{-} \pi^{0}\right)$ & GC & GR & Sgr dSph \\
\hline NFW Best & $1.3 \cdot 10^{-2}$ & $7.5 \cdot 10^{-3}$ & $5.2 \cdot 10^{-2}$ \\
Einasto Best & $3.0 \cdot 10^{-2}$ & $7.9 \cdot 10^{-3}$ & -
\end{tabular}

Table 6: Bounds from gamma rays on the branching fractions of $a \rightarrow \gamma \gamma$ and $a \rightarrow \pi^{+} \pi^{-} \pi^{0}$ in the minimal axion portal $\left(a \rightarrow \mu^{+} \mu^{-}\right)$. These are obtained neglecting all other sources of gamma rays and correspond to the best fit values for $m_{\mathrm{DM}}$ and $B$ and the propagation model giving smallest $\chi^{2}$. The bounds assume an equal boost factor for $e^{ \pm}$and gamma rays, and should be multiplied by $B_{e, \text { astro }} / B_{\gamma, \text { astro }}$ if the boost factors differ.

\begin{tabular}{c|ccc}
$\operatorname{Br}\left(a_{\ell} \rightarrow \gamma \gamma\right)$ & GC & GR & Sgr dSph \\
\hline NFW Best & $8.2 \cdot 10^{-3}$ & $4.6 \cdot 10^{-3}$ & $6.5 \cdot 10^{-2}$ \\
Einasto Best & $1.8 \cdot 10^{-2}$ & $4.9 \cdot 10^{-3}$ & -
\end{tabular}

Table 7: The same as Table 6, but for the leptonic axion portal $\left(a_{\ell} \rightarrow e^{+} e^{-}\right)$. The $a \rightarrow \pi^{+} \pi^{-} \pi^{0}$ mode is irrelevant in this case.

appreciable gamma ray or neutrino fluxes from the galactic center unless a very peaked dark matter halo profile is assumed. If the PAMELA/ATIC data is indicative of dark matter annihilation, however, then the galactic annihilation rate must be boosted by $O(1000)$. This large boost factor considerably enhances the potential for galactic gamma ray and neutrino signals from the dark sector. In this context, hints from the WMAP Haze may also point towards an annihilation explanation of PAMELA/ATIC.

In this paper, we have explored the robustness of dark matter annihilation predictions by considering cascade scenarios where dark matter annihilates into new resonances that in turn decay in one or more steps into standard model leptons. These cascade annihilation scenarios are directly motivated by the PAMELA/ATIC data, since light resonances can enhance the galactic annihilation rate through nonperturbative effects and explain the lepton-richness of the annihilation through kinematic thresholds.

We have shown that electron and muon cascades give reasonable fits to the PAMELA/ATIC data. As a rule of thumb, the best fit dark matter mass and boost factor both scale as $2^{n}$ for $n$-step cascade decays. We then compared these best fit values to constraints from gamma rays and neutrinos. The gamma ray bounds from FSR can be weakened by an order of magnitude through cascade decays, although increasing the length of cascades does not further weaken the bounds. Neutrino bounds for dark matter annihilating into muons are robust to changing the 
length of the cascade, which is particularly relevant for models with large branching fractions to muons such as the minimal axion portal.

Assuming standard NFW or Einasto halo profiles, there is tension between a dark matter annihilation interpretation of PAMELA/ATIC and the non-observation of galactic gamma rays or neutrinos. Such tension does not invalidate a dark matter annihilation hypothesis since there is considerable uncertainty in the dark matter halo distribution and velocity profile, and the constraints are uniformly weaker for shallower halo profiles. For gamma rays in particular, the galactic center and galactic ridge constraints assume an understanding of the dark matter halo profile in the inner $100 \mathrm{pc}$ of the galaxy, where there is considerable uncertainty. The dark matter halo profile in the inner $4^{\prime \prime}$ of the galaxy is even more uncertain, so we do not consider radio measurements of synchrotron to be constraining. Also, for both gamma rays and neutrinos, the bounds can be weakened if the astrophysical boost factor for electrons/positrons is larger than those for gamma rays and neutrinos.

If a dark matter annihilation scenario is realized in nature with the boost factor suggested by PAMELA/ATIC, then one would expect future experiments to see a gamma ray or neutrino flux given standard halo assumptions. ANTARES [56], IceCube [57, and KM3NeT [58] will greatly increase current sensitivity to upward-going muons resulting from galactic neutrinos. Future atmospheric Cerenkov telescopes as envisioned in Ref. [59] will also improve the prospects of finding gamma rays from dark matter annihilation. While we did not include the effect of ICS in our gamma ray analysis, ICS is expected to be a dominant dark matter annihilation signal in the energy range available to the Fermi Gamma-ray Space Telescope [60]. Ultimately, one hopes that future experiments could probe the detailed energy spectra of dark matter annihilation products to distinguish between direct annihilation and the cascade scenarios considered here.

Note added: While completing this paper, we became aware of Ref. 61] which considers similar issues.

\section{Acknowledgments}

We thank Shantanu Desai from the Super-K collaboration for providing us with $95 \%$ confidence bounds on the upward-going muon flux. This work was supported in part by the Director, Office of Science, Office of High Energy and Nuclear Physics, of the US Department of Energy under Contract DE-AC02-05CH11231, and in part by the National Science Foundation under grant PHY-0457315. The work of Y.N. was supported by the National Science Foundation under grant PHY-0555661, by a DOE OJI, and by the Alfred P. Sloan Foundation. D.S. is supported by the National Science Foundation, and J.T. is supported by the Miller Institute for Basic Research in Science. 


\section{A Cascade Energy Spectra}

In this appendix, we present formulae for the energy spectra used in the text. In general, the energy spectra of final state particles in cascade annihilations are functions of all the intermediate masses and helicities. In the limit of large mass hierarchies and scalar decays, however, the energy spectra greatly simplify, and we use these simplified formulae in our analysis.

Consider cascading fields $\phi_{i}$ of mass $m_{i}\left(m_{i+1}>2 m_{i}\right)$ and a final state $\psi$ with mass $m_{\psi}$. Cascade annihilation occurs through $\phi_{i+1} \rightarrow \phi_{i} \phi_{i}(i=1,2, \cdots)$, and in the last stage, $\phi_{1}$ decays into $\psi+X$. Let the energy of $\psi$ in the $\phi_{1}$ rest frame be $E_{0}$. Defining

$$
x_{0}=\frac{2 E_{0}}{m_{1}}, \quad \epsilon_{0}=\frac{2 m_{\psi}}{m_{1}}
$$

the $\psi$ energy spectrum is a function of $x_{0}$ and $\epsilon_{0}$

$$
\frac{d \tilde{N}_{\psi}}{d x_{0}}=\frac{d \tilde{N}_{\psi}}{d x_{0}}\left(x_{0}, \epsilon_{0}\right)
$$

where $\epsilon_{0} \leq x_{0} \leq 1$. In the case where dark matter $\chi$ annihilates directly into $\psi+X$, we can regard $\phi_{1}$ as the initial state of dark matter annihilation, $\chi \chi$. In this case $d \tilde{N}_{\psi} / d x_{0}$ is the primary injection spectrum with $m_{1}=2 m_{\mathrm{DM}}$.

Now consider the previous step in the cascade annihilation, $\phi_{2} \rightarrow \phi_{1} \phi_{1}$, with one of the $\phi_{1}$ decaying into $\psi+X$. Let the energy of $\psi$ in the $\phi_{2}$ rest frame be $E_{1}$ and define

$$
x_{1}=\frac{2 E_{1}}{m_{2}}, \quad \epsilon_{1}=\frac{2 m_{1}}{m_{2}} .
$$

Assuming isotropic scalar decays, the $\psi$ energy spectrum in the $\phi_{2}$ rest frame is

$$
\frac{d \tilde{N}_{\psi}}{d x_{1}}=\int_{-1}^{1} d \cos \theta \int_{\epsilon_{0}}^{1} d x_{0} \frac{d \tilde{N}_{\psi}}{d x_{0}} \delta\left(2 x_{1}-x_{0}-\cos \theta \sqrt{x_{0}^{2}-\epsilon_{0}^{2}} \sqrt{1-\epsilon_{1}^{2}}\right)
$$

where $\theta$ is the angle between the $\psi$ momentum and the $\phi_{1}$ boost axis as measured in the $\phi_{1}$ rest frame.

Equation (27) is complicated to solve in general, but in the limit $\epsilon_{i} \rightarrow 0(i=0,1, \cdots)$, it reduces to a simple convolution:

$$
\frac{d \tilde{N}_{\psi}}{d x_{1}}=\int_{x_{1}}^{1} \frac{d x_{0}}{x_{0}} \frac{d \tilde{N}_{\psi}}{d x_{0}}+\mathcal{O}\left(\epsilon_{i}^{2}\right)
$$

where $0 \leq x_{1} \leq 1$ up to $\mathcal{O}\left(\epsilon_{i}^{2}\right)$ effects. This convolution can be iterated as many times as necessary to build up the desired energy spectrum for an $n$-step cascade decay:

$$
\frac{d \tilde{N}_{\psi}}{d x_{n}}=\int_{x_{n}}^{1} \frac{d x_{n-1}}{x_{n-1}} \frac{d \tilde{N}_{\psi}}{d x_{n-1}}+\mathcal{O}\left(\epsilon_{i}^{2}\right)
$$


where $x_{n-1}=2 E_{n-1} / m_{n}$ with $E_{n-1}$ being the energy of $\psi$ in the $\phi_{n}$ rest frame, and $0 \leq x_{n} \leq 1$ up to $\mathcal{O}\left(\epsilon_{i}^{2}\right)$ effects. Note that we here adopt the normalization convention of

$$
\int_{0}^{1} d x_{n} \frac{d \tilde{N}_{\psi}}{d x_{n}}=1
$$

regardless of the value of $n$, so that the injection spectra per dark matter annihilation must be multiplied by the multiplicity of $\psi$ in the final state.

\section{A.1 Direct electron spectra}

Here we derive the spectra of electrons/positrons arising directly from $\phi_{1}$ decay, $\phi_{1} \rightarrow e^{+} e^{-}$(or dark matter annihilation, $\chi \chi \rightarrow e^{+} e^{-}$). Ignoring the effect of final state radiation to smooth the spectrum, the electron energy spectrum is given by

$$
\frac{d \tilde{N}_{e}}{d x_{0}}=\delta\left(1-x_{0}\right)
$$

where we have adopted the convention $\int_{0}^{1} d x_{0} \delta\left(1-x_{0}\right)=1$. The positron energy spectrum is identical.

Applying the simplified convolution formula in Eq. (29) for an $n$-step cascade annihilation, we then find

$$
\begin{aligned}
& \frac{d \tilde{N}_{e}}{d x_{1}}=1, \\
& \frac{d \tilde{N}_{e}}{d x_{2}}=\ln \frac{1}{x_{2}}, \\
& \frac{d \tilde{N}_{e}}{d x_{n}}=Q_{n}\left(x_{n}\right),
\end{aligned}
$$

where we have defined

$$
Q_{n}(x) \equiv \frac{1}{(n-1) !}\left(\ln \frac{1}{x}\right)^{n-1} .
$$

Note that these are energy spectra for one of the electrons (or positrons), so that the electron (or positron) injection spectra per dark matter annihilation $\chi \chi \rightarrow 2 \phi_{n} \rightarrow \cdots \rightarrow 2^{n}\left(e^{+} e^{-}\right)$are

$$
\frac{d N_{e}}{d x_{n}}=2^{n} \frac{d \tilde{N}_{e}}{d x_{n}},
$$

where $x_{n}=E_{e} / m_{\mathrm{DM}}$ with $E_{e}$ being the electron (positron) energy in the center-of-mass frame for dark matter annihilation. The direct annihilation case, $\chi \chi \rightarrow e^{+} e^{-}$, corresponds to $n=0$. 


\section{A.2 Electron and neutrino spectra from muon decay}

Here we discuss the spectra of electrons, positrons and neutrinos arising from muon decay. Consider $\phi_{1} \rightarrow \mu^{+} \mu^{-}$(or $\chi \chi \rightarrow \mu^{+} \mu^{-}$) followed by $\mu \rightarrow e \nu_{e} \nu_{\mu}$. (One of the neutrinos here should be an anti-neutrino. We omit the particle-antiparticle identification here and below.) Assuming a massless electron, the (unpolarized) spectra of electrons and neutrinos in the rest frame of the muon are

$$
\begin{aligned}
& \frac{d \tilde{N}_{\mu \rightarrow e}}{d x_{-1}}=\frac{d \tilde{N}_{\mu \rightarrow \nu_{\mu}}}{d x_{-1}}=6\left(x_{-1}\right)^{2}-4\left(x_{-1}\right)^{3}, \\
& \frac{d \tilde{N}_{\mu \rightarrow \nu_{e}}}{d x_{-1}}=12\left(x_{-1}\right)^{2}-12\left(x_{-1}\right)^{3},
\end{aligned}
$$

where we are using the notation $x_{-1}=2 E_{-1} / m_{\mu}$, with $E_{-1}$ being the energy in the muon rest frame.

Applying the cascade convolution for electrons and muon neutrinos

$$
\begin{aligned}
& \frac{d \tilde{N}_{\mu \rightarrow e}}{d x_{0}}=\frac{d \tilde{N}_{\mu \rightarrow \nu_{\mu}}}{d x_{0}}=\frac{5}{3}-3 x_{0}^{2}+\frac{4}{3} x_{0}^{3}, \\
& \frac{d \tilde{N}_{\mu \rightarrow e}}{d x_{1}}=\frac{d \tilde{N}_{\mu \rightarrow \nu_{\mu}}}{d x_{1}}=-\frac{19}{18}+\frac{3}{2} x_{1}^{2}-\frac{4}{9} x_{1}^{3}+\frac{5}{3} Q_{2}\left(x_{1}\right), \\
& \frac{d \tilde{N}_{\mu \rightarrow e}}{d x_{2}}=\frac{d \tilde{N}_{\mu \rightarrow \nu_{\mu}}}{d x_{2}}=\frac{65}{108}-\frac{3}{4} x_{2}^{2}+\frac{4}{27} x_{2}^{3}-\frac{19}{18} Q_{2}\left(x_{2}\right)+\frac{5}{3} Q_{3}\left(x_{2}\right),
\end{aligned}
$$

and for electron neutrinos

$$
\begin{aligned}
& \frac{d \tilde{N}_{\mu \rightarrow \nu_{e}}}{d x_{0}}=2-6 x_{0}^{2}+4 x_{0}^{3}, \\
& \frac{d \tilde{N}_{\mu \rightarrow \nu_{e}}}{d x_{1}}=-\frac{5}{3}+3 x_{1}^{2}-\frac{4}{3} x_{1}^{3}+2 Q_{2}\left(x_{1}\right), \\
& \frac{d \tilde{N}_{\mu \rightarrow \nu_{e}}}{d x_{2}}=\frac{19}{18}-\frac{3}{2} x_{2}^{2}+\frac{4}{9} x_{2}^{3}-\frac{5}{3} Q_{2}\left(x_{2}\right)+2 Q_{3}\left(x_{2}\right) .
\end{aligned}
$$

Again, these are energy spectra for one of the electrons, positrons or (anti-)neutrinos. To obtain the injection spectra per dark matter annihilation, we must multiply the multiplicity factor, $2^{n}$ for $n$-step, and set $x_{n}=E / m_{\mathrm{DM}}$. Here, $E$ is the energy of a particle in the center-of-mass frame for dark matter annihilation.

For comparison, the corresponding formulae in the approximation of isotropic three-body decays are

$$
\begin{aligned}
& \frac{d \tilde{N}_{\mu \rightarrow e}}{d x_{-1}} \simeq 2 x_{-1} \\
& \frac{d \tilde{N}_{\mu \rightarrow e}}{d x_{n}} \simeq(-1)^{n+1}\left(2 x_{n}+2 \sum_{i=1}^{n+1}(-1)^{i} Q_{i}\left(x_{n}\right)\right) \equiv \bar{Q}_{n+1}\left(x_{n}\right) .
\end{aligned}
$$


As a rough rule of thumb, the electron spectrum for an $n$-step muon cascade has a shape between $(n+1)$ - and $(n+2)$-step electron cascades.

\section{A.3 Gamma ray spectra from final state radiation}

Primary gamma rays come from final state radiation in the decay $\phi_{1} \rightarrow \ell^{+} \ell^{-} \gamma$. In principle, one could do an exact calculation to $\mathcal{O}\left(\alpha_{\mathrm{EM}}\right)$ of the gamma ray spectrum, which would have the full $\epsilon_{0} \equiv 2 m_{\ell} / m_{1}$ dependence. Since we are using the simplified convolution formula in Eq. (29), it is not consistent to keep $\mathcal{O}\left(\epsilon_{0}^{2}\right)$ corrections in the exact gamma ray calculation, and it suffices to use twice the Altarelli-Parisi splitting formula

$$
\frac{d \tilde{N}_{\gamma}}{d x_{0}}=\frac{\alpha_{\mathrm{EM}}}{\pi} \frac{1+\left(1-x_{0}\right)^{2}}{x_{0}}\left\{-1+\ln \left(\frac{4\left(1-x_{0}\right)}{\epsilon_{0}^{2}}\right)\right\},
$$

where the normalization of $\tilde{N}_{\gamma}$ is such that $\int d x_{n} d \tilde{N}_{\gamma} / d x_{n}$ gives the (average) number of photons per $\phi_{1}$ decay. Note that the expression of Eq. (47) becomes negative at $x_{0}>1-e \epsilon_{0}^{2} / 4$, which does not correspond to the kinematic threshold. The error from this, however, is formally an $\mathcal{O}\left(\epsilon_{0}^{2}\right)$ effect.

Applying the simplified convolution formula, we obtain

$$
\begin{aligned}
& \frac{d \tilde{N}_{\gamma}}{d x_{1}}=\frac{\alpha_{\mathrm{EM}}}{\pi} \frac{1}{x_{1}}\left\{\left(-1+\ln \frac{4}{\epsilon_{0}^{2}}\right) R_{1}\left(x_{1}\right)+S_{1}\left(x_{1}\right)\right\}, \\
& \frac{d \tilde{N}_{\gamma}}{d x_{2}}=\frac{\alpha_{\mathrm{EM}}}{\pi} \frac{1}{x_{2}}\left\{\left(-1+\ln \frac{4}{\epsilon_{0}^{2}}\right) R_{2}\left(x_{2}\right)+S_{2}\left(x_{2}\right)\right\},
\end{aligned}
$$

where

$$
\begin{aligned}
R_{1}(x)= & 2-x-x^{2}+2 x \ln x \\
R_{2}(x)= & 2-3 x+x^{2}+x \ln x-x(\ln x)^{2}, \\
S_{1}(x)= & \left(\frac{\pi^{2}}{3}-1\right) x+x^{2}+2 x \ln x+\left(2-x-x^{2}\right) \ln (1-x)-2 x \operatorname{Li}_{2}(x), \\
S_{2}(x)= & \left(\frac{\pi^{2}}{6}+2-2 \zeta(3)\right) x-2 x^{2}-\left(\frac{\pi^{2}}{3}-3\right) x \ln x+\left(2-3 x+x^{2}\right) \ln (1-x) \\
& -x(\ln x)^{2}-x \operatorname{Li}_{2}(x)+2 x \operatorname{Li}_{3}(x) .
\end{aligned}
$$

The photon injection spectra per dark matter annihilation are then given by

$$
\frac{d N_{\gamma}}{d x_{n}}=2^{n} \frac{d \tilde{N}_{\gamma}}{d x_{n}}
$$

where $x_{n}=E_{\gamma} / m_{\mathrm{DM}}$, with $E_{\gamma}$ being the photon energy in the center-of-mass frame for dark matter annihilation. Here, $\epsilon_{0}=m_{\ell} / m_{\mathrm{DM}}$ for direct annihilation and $\epsilon_{0}=2 m_{\ell} / m_{1}$ otherwise. 
For the hardest gamma rays near $x_{n} \rightarrow 1$, the behavior of $d \tilde{N}_{\gamma} / d x_{n}$ is

$$
\begin{aligned}
& \frac{d \tilde{N}_{\gamma}}{d x_{0}} \simeq \frac{\alpha_{\mathrm{EM}}}{\pi} \frac{1}{x_{0}} \ln \left(\frac{4\left(1-x_{0}\right)}{\epsilon_{0}^{2}}\right) \\
& \frac{d \tilde{N}_{\gamma}}{d x_{1}} \simeq \frac{\alpha_{\mathrm{EM}}}{\pi} \frac{1-x_{1}}{x_{1}} \ln \left(\frac{4\left(1-x_{1}\right)}{\epsilon_{0}^{2}}\right), \\
& \frac{d \tilde{N}_{\gamma}}{d x_{2}} \simeq \frac{\alpha_{\mathrm{EM}}}{\pi} \frac{\left(1-x_{2}\right)^{2}}{2 x_{2}} \ln \left(\frac{4\left(1-x_{2}\right)}{\epsilon_{0}^{2}}\right) .
\end{aligned}
$$

Compared to direct annihilation into leptons, a 1-step cascade annihilation gives a gamma ray spectrum that is suppressed not only by $\ln \left(2 m_{\ell} / m_{1}\right) / \ln \left(m_{\ell} / m_{\mathrm{DM}}\right)$ but also by an additional suppression factor of $(1-x)$ for the highest energy gamma rays.

\section{A.4 Gamma ray subtlety for muons}

There are actually two contributions to the gamma ray spectrum for $\phi_{1} \rightarrow \mu^{+} \mu^{-} \gamma$. In addition to final state radiation from muons, there is the radiative decay of the muon $\mu \rightarrow e \nu_{e} \nu_{\mu} \gamma$. Formally, this contribution is suppressed by a factor of $1 / \ln \left(m_{\mu} / m_{1}\right)$ or $\left(1-x_{n}\right)^{2}$, but for $m_{1} \approx m_{\mu}$, it is an important effect.

The gamma ray spectrum in the muon rest frame is known in the limit that $r=m_{e}^{2} / m_{\mu}^{2}$ is small [62]. Assuming unpolarized muons, we can derive the 0 -step cascade annihilation spectrum from the muon rest frame spectrum

$$
\begin{aligned}
& \frac{d \tilde{N}_{\mu \rightarrow \gamma}}{d x_{-1}}=\frac{\alpha_{\mathrm{EM}}}{3 \pi} \frac{1}{x_{-1}}\left(T_{-1}\left(x_{-1}\right) \ln \frac{1}{r}+U_{-1}\left(x_{-1}\right)\right), \\
& \frac{d \tilde{N}_{\mu \rightarrow \gamma}}{d x_{0}}=\frac{\alpha_{\mathrm{EM}}}{3 \pi} \frac{1}{x_{0}}\left(T_{0}\left(x_{0}\right) \ln \frac{1}{r}+U_{0}\left(x_{0}\right)\right)
\end{aligned}
$$

where

$$
\begin{aligned}
T_{-1}(x)= & (1-x)\left(3-2 x+4 x^{2}-2 x^{3}\right), \\
T_{0}(x)= & 3+\frac{2}{3} x-6 x^{2}+3 x^{3}-\frac{2}{3} x^{4}+5 x \ln x, \\
U_{-1}(x)= & (1-x)\left(-\frac{17}{2}+\frac{23}{6} x-\frac{101}{12} x^{2}+\frac{55}{12} x^{3}+\left(3-2 x+4 x^{2}-2 x^{3}\right) \ln (1-x)\right), \\
U_{0}(x)= & -\frac{17}{2}-\frac{3}{2} x+\frac{191}{12} x^{2}-\frac{23}{3} x^{3}+\frac{7}{4} x^{4}+\left(3+\frac{2}{3} x-6 x^{2}+3 x^{3}-\frac{2}{3} x^{4}\right) \ln (1-x) \\
& -\frac{28}{3} x \ln x+5 x \ln (1-x) \ln x+5 x \operatorname{Li}_{2}(1-x) .
\end{aligned}
$$

The convolutions for 1- and 2-step decays are straightforward to derive. 
Note again that $\int d x_{n} d \tilde{N}_{\mu \rightarrow \gamma} / d x_{n}$ give the (average) number of photons per muon decay. The photon spectra from radiative muon decay per dark matter annihilation are then given by $2^{n} d \tilde{N}_{\mu \rightarrow \gamma} / d x_{n}$. The total photon injection spectra per dark matter annihilation are given by

$$
\frac{d N_{\gamma}}{d x_{n}}=2^{n}\left(\frac{d \tilde{N}_{\gamma}}{d x_{n}}+2 \frac{d \tilde{N}_{\mu \rightarrow \gamma}}{d x_{n}}\right)
$$

where $x_{n}=E_{\gamma} / m_{\mathrm{DM}}$, with $E_{\gamma}$ being the photon energy in the center-of-mass frame for dark matter annihilation.

\section{A.5 Rare modes in the axion portal}

In Section 6, we consider bounds on rare $a \rightarrow \gamma \gamma$ and $a \rightarrow \pi^{+} \pi^{-} \pi^{0}$ decay modes in axion portal models. The axion portal has both a 1-step and a 2-step component, and the gamma ray spectrum for each can be calculated straightforwardly. The $a \rightarrow \gamma \gamma$ spectra are identical (up to normalization) to the $\phi_{1} \rightarrow e^{+} e^{-}$spectra already calculated:

$$
\begin{aligned}
& \frac{d \tilde{N}_{a \rightarrow \gamma}}{d x_{1}}=2, \\
& \frac{d \tilde{N}_{a \rightarrow \gamma}}{d x_{2}}=2 \ln \frac{1}{x_{2}},
\end{aligned}
$$

where the normalization of $\tilde{N}_{a \rightarrow \gamma}$ is such that $\int d x_{n} d \tilde{N}_{a \rightarrow \gamma} / d x_{n}$ gives the number of photons per $a$ decay.

For $a \rightarrow \pi^{+} \pi^{-} \pi^{0}$ followed by $\pi^{0} \rightarrow \gamma \gamma$, we can use the $\bar{Q}_{n}$ function from Eq. (46) if we assume that $m_{a} \gg m_{\pi}$ and that the $a \rightarrow 3 \pi$ decay is isotropic:

$$
\begin{aligned}
& \frac{d \tilde{N}_{a \rightarrow \pi^{0} \rightarrow \gamma}}{d x_{1}} \simeq 2 \bar{Q}_{2}\left(x_{1}\right), \\
& \frac{d \tilde{N}_{a \rightarrow \pi^{0} \rightarrow \gamma}}{d x_{2}} \simeq 2 \bar{Q}_{3}\left(x_{2}\right),
\end{aligned}
$$

where again the normalization of $\tilde{N}_{a \rightarrow \pi^{0} \rightarrow \gamma}$ is such that $\int d x_{n} d \tilde{N}_{a \rightarrow \pi^{0} \rightarrow \gamma} / d x_{n}$ gives the number of photons per $a$ decay. One should keep in mind that $m_{a} \simeq 3 m_{\pi}$ in the region of interest, but the hierarchical cascade approximation is still reasonably representative of the true energy spectrum.

\section{B Leptonic Axion Portal}

In the minimal axion portal construction, the axion has large hadronic couplings, and is therefore strongly constrained by beam dump and rare meson decay experiments. In particular, the axion is forced to decay primarily into muons, and, as we saw in Section 5, there is some degree of 
tension between a muon annihilation scenario and the absence of galactic neutrinos. Also, we saw in Section 6 that there are strong gamma ray bounds on the $a \rightarrow \pi^{+} \pi^{-} \pi^{0}$ decay mode, which in the minimal axion portal arises from axion-pion mixing.

Since the decay properties of the axion are irrelevant for dark matter freezeout, we can easily modify the couplings of the axion to standard model fields without losing the good features of this scenario. In particular, we can construct a leptonic axion portal model where the axion has no hadronic couplings. While such a leptonic axion could decay into muons as in the minimal axion portal, in the text we consider the less constrained case where the leptonic axion decays primarily into electrons.

The simplest example for the leptonic axion portal can be constructed as follows. Vectorlike fermion dark matter $\psi / \psi^{c}$ obtains a mass from spontaneous symmetry breaking through the vacuum expectation value of a complex scalar $S_{\ell}$ :

$$
\mathcal{L}=-\xi S_{\ell} \psi \psi^{c}+\text { h.c. }, \quad S_{\ell}=\left(f_{a}+\frac{s_{\ell}}{\sqrt{2}}\right) e^{i a_{\ell} / \sqrt{2} f_{a}},
$$

where $a_{\ell}$ is the pseudoscalar axion, $s_{\ell}$ is a light scalar, and $f_{a}$ is the axion decay constant, which is assumed to be of order $\mathrm{TeV}$. As in the minimal axion portal, the masses of $s_{\ell}$ and $a_{\ell}$ can be considered as free parameters. In order for $a_{\ell}$ to decay into leptons, $S_{\ell}$ must be charged under a leptonic symmetry (which is softly broken to give a mass to $a_{\ell}$ ). This requires introducing separate electron-type and neutrino-type Higgses:

$$
\mathcal{L}=-\lambda_{e} \ell h_{e} e^{c}-\lambda_{\nu} \ell h_{\nu} \nu^{c}-A_{\ell} S_{\ell} h_{e} h_{\nu}+\text { h.c. }
$$

These interactions force the standard model leptons to carry axial leptonic charges. (Small neutrino masses can be obtained through the standard see-saw mechanism, and it is straightforward to extend the model to incorporate supersymmetry.)

In order to eliminate the hadronic couplings of $a_{\ell}$, the standard model quarks are assumed not to carry charges under the leptonic symmetry. This requires introducing one or more new Higgses for the quark sector, which must also be singlets under the leptonic symmetry. As long as the potential for these Higgses preserves the leptonic symmetry, then $a_{\ell}$ will have no hadronic couplings and cannot mix with the neutral mesons after the leptonic symmetry is spontaneously broken.

The absence of hadronic couplings allows $m_{a_{\ell}}$ to be lighter than $2 m_{\mu}$, and thus $a_{\ell}$ to decay dominantly into electrons while satisfying beam dump and rare meson decay constraints. Also, the leptonic axion $a_{\ell}$ has no $a_{\ell} \rightarrow \pi^{+} \pi^{-} \pi^{0}$ decay mode, reducing the gamma ray constraints in the case that $m_{a_{\ell}}>3 m_{\pi}$ (where $a_{\ell}$ dominantly decays into muons). Since the strongest astrophysical bounds on light degrees of freedom come from hadronic couplings [63], $m_{a_{\ell}}$ might even be as light as $2 m_{e}$, although a detailed study of the constraints on the leptonic axion is beyond the scope of this paper. 
As an example of the quark sector interactions, there could be separate up-type and downtype Higgses. In this case, it is natural to assume a hadronic symmetry under which the quarks carry axial charges:

$$
\mathcal{L}=-\lambda_{u} q h_{u} u^{c}-\lambda_{d} q h_{d} d^{c}-A_{q} S_{q} h_{u} h_{d}+\text { h.c. }
$$

The axion contained in the field $S_{q}$ could then be the QCD axion and solve the strong CP problem. To avoid astrophysical constraints, however, the vacuum expectation value of $S_{q}$ must be $\gtrsim 10^{10} \mathrm{GeV}$, much larger than $\left\langle S_{\ell}\right\rangle=O(\mathrm{TeV})$. Explicit breaking of the hadronic symmetry must also be much smaller than that of the leptonic symmetry.

\section{References}

[1] O. Adriani et al., arXiv:0810.4995 [astro-ph].

[2] J. Chang et al., Nature 456, 362 (2008).

[3] See, for example, D. Hooper, P. Blasi and P. D. Serpico, arXiv:0810.1527 [astroph]; H. Yüksel, M. D. Kistler and T. Stanev, arXiv:0810.2784 [astro-ph]; S. Profumo, arXiv:0812.4457 [astro-ph]; K. Ioka, arXiv:0812.4851 [astro-ph]; H- B. Hu, Q. Yuan, B. Wang, C. Fan, J.-L. Zhang and X.-J. Bi, arXiv:0901.1520 [astro-ph].

[4] See, for example, P.-f. Yin, Q. Yuan, J. Liu, J. Zhang, X.-j. Bi, S.-h. Zhu and X. Zhang, arXiv:0811.0176 [hep-ph]; C.-R. Chen, M. M. Nojiri, F. Takahashi and T. T. Yanagida, arXiv:0811.3357 [astro-ph]; E. Nardi, F. Sannino and A. Strumia, arXiv:0811.4153 [hep-ph]; K. Ishiwata, S. Matsumoto and T. Moroi, arXiv:0811.4492 [astro-ph]; arXiv:0811.0250 [hepph]; A. Arvanitaki, S. Dimopoulos, S. Dubovsky, P. W. Graham, R. Harnik and S. Rajendran, arXiv:0812.2075 [hep-ph]; K. Hamaguchi, S. Shirai and T. T. Yanagida, arXiv:0812.2374 [hep-ph]; F. Takahashi and E. Komatsu, arXiv:0901.1915 [astro-ph].

[5] M. Cirelli, M. Kadastik, M. Raidal and A. Strumia, arXiv:0809.2409 [hep-ph].

[6] N. Arkani-Hamed, D. P. Finkbeiner, T. Slatyer and N. Weiner, arXiv:0810.0713 [hep-ph].

[7] Y. Nomura and J. Thaler, arXiv:0810.5397 [hep-ph].

[8] I. Cholis, G. Dobler, D. P. Finkbeiner, L. Goodenough and N. Weiner, arXiv:0811.3641 [astro-ph].

[9] See, for example, M. Fairbairn and J. Zupan, arXiv:0810.4147 [hep-ph]; A. E. Nelson and C. Spitzer, arXiv:0810.5167 [hep-ph]; R. Harnik and G. D. Kribs, arXiv:0810.5557 [hepph]; Y. Bai and Z. Han, arXiv:0811.0387 [hep-ph]; P. J. Fox and E. Poppitz, arXiv:0811.0399 [hep-ph]; K. M. Zurek, arXiv:0811.4429 [hep-ph]; E. J. Chun and J. C. Park, arXiv:0812.0308 [hep-ph]; R. Allahverdi, B. Dutta, K. Richardson-McDaniel and Y. Santoso, arXiv:0812.2196 
[hep-ph]; D. Hooper, A. Stebbins and K. M. Zurek, arXiv:0812.3202 [hep-ph]; C.-R. Chen, K. Hamaguchi, M. M. Nojiri, F. Takahashi and S. Torii, arXiv:0812.4200 [astro-ph]; I. Gogoladze, R. Khalid, Q. Shafi and H. Yüksel, arXiv:0901.0923 [hep-ph].

[10] D. P. Finkbeiner, Astrophys. J. 614, 186 (2004) arXiv:astro-ph/0311547]; G. Dobler and D. P. Finkbeiner, Astrophys. J. 680, 1222 (2008) [arXiv:0712.1038 [astro-ph]].

[11] D. P. Finkbeiner, arXiv:astro-ph/0409027; D. Hooper, D. P. Finkbeiner and G. Dobler, Phys. Rev. D 76, 083012 (2007) arXiv:0705.3655 [astro-ph]].

[12] I. Cholis, L. Goodenough and N. Weiner, arXiv:0802.2922 [astro-ph].

[13] J. Zhang, X.-j. Bi, J. Liu, S.-M. Liu, P.-f. Yin, Q. Yuan and S.-h. Zhu, arXiv:0812.0522 [astro-ph].

[14] N. F. Bell and T. D. Jacques, arXiv:0811.0821 [astro-ph].

[15] G. Bertone, M. Cirelli, A. Strumia and M. Taoso, arXiv:0811.3744 [astro-ph].

[16] L. Bergström, G. Bertone, T. Bringmann, J. Edsjö and M. Taoso, arXiv:0812.3895 [astro-ph].

[17] J. Hisano, M. Kawasaki, K. Kohri and K. Nakayama, arXiv:0812.0219 [hep-ph].

[18] J. Liu, P.-f. Yin and S.-h. Zhu, arXiv:0812.0964 [astro-ph].

[19] O. Adriani et al., arXiv:0810.4994 [astro-ph].

[20] F. Aharonian et al., Phys. Rev. Lett. 97, 221102 (2006) [Erratum-ibid. 97, 249901 (2006)] arXiv:astro-ph/0610509].

[21] F. Aharonian et al., Nature 439, 695 (2006) [arXiv:astro-ph/0603021].

[22] F. Aharonian et al., Astropart. Phys. 29, 55 (2008) [arXiv:0711.2369 [astro-ph]].

[23] J. Hisano, S. Matsumoto and M. M. Nojiri, Phys. Rev. Lett. 92, 031303 (2004) arXiv:hep-ph/0307216]; J. Hisano, S. Matsumoto, M. M. Nojiri and O. Saito, Phys. Rev. D 71, 063528 (2005) arXiv:hep-ph/0412403; M. Cirelli, A. Strumia and M. Tamburini, Nucl. Phys. B 787, 152 (2007) arXiv:0706.4071 [hep-ph]]; J. March-Russell, S. M. West, D. Cumberbatch and D. Hooper, JHEP 0807, 058 (2008) arXiv:0801.3440 [hep-ph]]; M. Lattanzi and J. Silk, arXiv:0812.0360 [astro-ph].

[24] M. Pospelov and A. Ritz, arXiv:0810.1502 [hep-ph]; J. March-Russell and S. M. West, arXiv:0812.0559 [astro-ph]; W. Shepherd, T. M. P. Tait and G. Zaharijas, arXiv:0901.2125 [hep-ph].

[25] S. Desai et al. [Super-Kamiokande Collaboration], Phys. Rev. D 70, 083523 (2004) [Erratumibid. D 70, 109901 (2004)] [arXiv:hep-ex/0404025].

[26] M. Pospelov, A. Ritz and M. Voloshin, Phys. Lett. B 662, 53 (2008) arXiv:0711.4866 [hep$\mathrm{ph}]$. 
[27] D. P. Finkbeiner and N. Weiner, Phys. Rev. D 76, 083519 (2007) arXiv:astro-ph/0702587.

[28] P. Picozza et al., Astropart. Phys. 27, 296 (2007) arXiv:astro-ph/0608697.

[29] T. G. Guzik et al., Adv. Space Res. 33, 1763 (2004).

[30] T. Delahaye, R. Lineros, F. Donato, N. Fornengo and P. Salati, Phys. Rev. D 77, 063527 (2008) arXiv:0712.2312 [astro-ph]].

[31] J. N. Bahcall and R. M. Soneira, Astrophys. J. Suppl. 44, 73 (1980).

[32] J. F. Navarro, C. S. Frenk and S. D. M. White, Astrophys. J. 490, 493 (1997) arXiv:astro-ph/9611107].

[33] J. F. Navarro et al., Mon. Not. Roy. Astron. Soc. 349, 1039 (2004) arXiv:astro-ph/0311231.

[34] J. Hisano, S. Matsumoto, O. Saito and M. Senami, Phys. Rev. D 73, 055004 (2006) arXiv:hep-ph/0511118.

[35] J. Lavalle, J. Pochon, P. Salati and R. Taillet, arXiv:astro-ph/0603796.

[36] I. V. Moskalenko and A. W. Strong, Astrophys. J. 493, 694 (1998) arXiv:astro-ph/9710124, as parameterized in E. A. Baltz and J. Edsjö, Phys. Rev. D 59, 023511 (1999) arXiv:astro-ph/9808243.

[37] High Energy Stereoscopic System (H.E.S.S.), http://www.mpi-hd.mpg.de/hfm/HESS/

[38] F. Aharonian et al., Phys. Rev. Lett. 101, 261104 (2008) arXiv:0811.3894 [astro-ph]].

[39] A. Birkedal, K. T. Matchev, M. Perelstein and A. Spray, arXiv:hep-ph/0507194.

[40] G. D. Mack, T. D. Jacques, J. F. Beacom, N. F. Bell and H. Yüksel, Phys. Rev. D 78, 063542 (2008) arXiv:0803.0157 [astro-ph]].

[41] N. W. Evans, F. Ferrer and S. Sarkar, Phys. Rev. D 69, 123501 (2004) arXiv:astro-ph/0311145.

[42] R. D. Davies, D. Walsh and R. S. Booth, Mon. Not. Roy. Astron. Soc. 177, 319 (1976).

[43] A. A. Dutton, F. C. van den Bosch, A. Dekel and S. Courteau, Astrophys. J. 654, 27 (2006) arXiv:astro-ph/0604553.

[44] D. Merritt, M. Milosavljević, L. Verde and R. Jimenez, Phys. Rev. Lett. 88, 191301 (2002) arXiv:astro-ph/0201376].

[45] J. Diemand, M. Kuhlen, P. Madau, M. Zemp, B. Moore, D. Potter and J. Stadel, arXiv:0805.1244 [astro-ph]; J. F. Navarro et al., arXiv:0810.1522 [astro-ph].

[46] E. Borriello, A. Cuoco and G. Miele, arXiv:0809.2990 [astro-ph]; arXiv:0812.2932 [astro-ph].

[47] A. de Oliveira-Costa, M. Tegmark, B. M. Gaensler, J. Jonas, T. L. Landecker and P. Reich, arXiv:0802.1525 [astro-ph]. 
[48] S. Ritz and D. Seckel, Nucl. Phys. B 304, 877 (1988).

[49] C. Amsler et al. [Particle Data Group], Phys. Lett. B 667, 1 (2008).

[50] V. Barger, W.-Y. Keung, G. Shaughnessy and A. Tregre, Phys. Rev. D 76, 095008 (2007) arXiv:0708.1325 [hep-ph]].

[51] H. L. Lai et al., Eur. Phys. J. C 12, 375 (2000) arXiv:hep-ph/9903282].

[52] S. Fukuda et al., Nucl. Instrum. Meth. A 501, 418 (2003).

[53] S. Desai, private communication.

[54] M. Kamionkowski and S. Profumo, Phys. Rev. Lett. 101, 261301 (2008) arXiv:0810.3233 [astro-ph]].

[55] Y. B. Zel'dovich, A. A. Klypin, M. Y. Khlopov and V. M. Chechetkin, Sov. J. Nucl. Phys. 31, 664 (1980) [Yad. Fiz. 31, 1286 (1980)].

[56] E. Aslanides et al., arXiv:astro-ph/9907432.

[57] J. Ahrens et al., Astropart. Phys. 20, 507 (2004) arXiv:astro-ph/0305196].

[58] A. Kappes, arXiv:0711.0563 [astro-ph].

[59] J. Buckley et al., arXiv:0810.0444 [astro-ph].

[60] Fermi Gamma-ray Space Telescope (formerly GLAST), http://fermi.gsfc.nasa.gov/

[61] P. Meade, M. Papucci and T. Volansky, arXiv:0901.2925 [hep-ph].

[62] Y. Kuno and Y. Okada, Rev. Mod. Phys. 73, 151 (2001) arXiv:hep-ph/9909265.

[63] G. G. Raffelt, Phys. Rept. 198, 1 (1990). 